ARTICLE

\title{
Polyunsaturated fatty acids and p38-MAPK link metabolic reprogramming to cytoprotective gene expression during dietary restriction
}

\author{
Manish Chamoli 10 1,2,6, Anita Goyala1,6, Syed Shamsh Tabrez 1,3,6, Atif Ahmed Siddiqui ${ }^{1}$, Anupama Singh (10 1, \\ Adam Antebi ${ }^{3,4}$, Gordon J. Lithgow ${ }^{2}$, Jennifer L. Watts ${ }^{5} \&$ Arnab Mukhopadhyay (i) $^{1 凶}$
}

The metabolic state of an organism instructs gene expression modalities, leading to changes in complex life history traits, such as longevity. Dietary restriction (DR), which positively affects health and life span across species, leads to metabolic reprogramming that enhances utilisation of fatty acids for energy generation. One direct consequence of this metabolic shift is the upregulation of cytoprotective (CyTP) genes categorized in the Gene Ontology (GO) term of "Xenobiotic Detoxification Program" (XDP). How an organism senses metabolic changes during nutritional stress to alter gene expression programs is less known. Here, using a genetic model of DR, we show that the levels of polyunsaturated fatty acids (PUFAs), especially linoleic acid (LA) and eicosapentaenoic acid (EPA), are increased following DR and these PUFAs are able to activate the CyTP genes. This activation of CyTP genes is mediated by the conserved p38 mitogen-activated protein kinase (p38-MAPK) pathway. Consequently, genes of the PUFA biosynthesis and p38-MAPK pathway are required for multiple paradigms of DR-mediated longevity, suggesting conservation of mechanism. Thus, our study shows that PUFAs and p38-MAPK pathway function downstream of DR to help communicate the metabolic state of an organism to regulate expression of CyTP genes, ensuring extended life span.

\footnotetext{
${ }^{1}$ Molecular Aging Laboratory, National Institute of Immunology, Aruna Asaf Ali Marg, New Delhi 110067, India. ${ }^{2}$ Buck Institute for Research on Aging, 8001 Redwood Blvd., Novato, CA 94945, USA. ${ }^{3}$ Department of Molecular Genetics of Ageing, Max Planck Institute for Biology of Ageing, Cologne 50931, Germany. ${ }^{4}$ Cologne Excellence Cluster on Cellular Stress Responses in Aging Associated Diseases, University of Cologne, Cologne 50931, Germany. ${ }^{5}$ School of Molecular Biosciences, Washington State University, Pullman, WA 99164-7520, USA. ${ }^{6}$ These authors contributed equally: Manish Chamoli, Anita Goyala, Syed Shamsh Tabrez. ${ }^{凶}$ email: arnab@nii.ac.in
} 
D ietary restriction (DR) is the only non-genetic manipulation that extends life span and delays age-associated diseases in most model systems. In non-human primates, DR has been shown to delay age-onset type 2 diabetes, cardiovascular diseases and loss of cognitive abilities ${ }^{1}$. Although the molecular mechanisms of DR are less clear, it is associated with dramatic reprogramming of metabolism that shifts from fat storage to enhanced fatty acid utilization, influencing cellular stress signaling pathways and reactive oxygen species (ROS) production $^{1-7}$. Using C. elegans, we have shown that this metabolic reprogramming leads to the upregulation of the cytoprotective (CyTP) genes categorized to function in the xenobiotic detoxification program (XDP), based on Gene Ontology (GO) terms, and this coupling is essential for life-span extension during $\mathrm{DR}^{3}$. Knocking down either the central regulator of fatty acid metabolism (NHR-49) or transcription factors (NHR-8 or PHA-4) required for the expression of CyTP genes suppresses DRinduced longevity ${ }^{3}$. However, the signaling events that lead to activation of CyTP genes following increased fatty acid oxidation are not known.

Mitogen-activated protein kinases (MAPK) constitute the evolutionarily conserved signaling cascades that transduce signals, generated by extracellular stimuli, to the nucleus. The three wellcharacterized subfamilies of MAP kinases which have representatives in all eukaryotes include ERKs (extracellular signalregulated kinases), SAPK/JNKs (stress-activated protein kinases/ c-Jun N-terminal kinases) and $\mathrm{p} 38-\mathrm{MAPK}^{8}$. The p38-MAPK pathway has been implicated in diverse cellular responses including inflammation, stress response, cell cycle, apoptosis, development, differentiation, senescence, and tumorigenesis ${ }^{9-12}$. In C. elegans, the p38-MAPK pathway is an important regulator of innate immunity, oxidative stress resistance, neuronal development as well as longevity ${ }^{13-17}$. Upon exposure to pathogens or oxidative stress, a cascade of signaling events involving NSY-1 (MAPKKK)-SEK-1 (MAPKK) activates the downstream effector kinase PMK-1/p38-MAPK. This in turn regulates the transcriptional activation of innate immunity or ROS detoxification genes, respectively ${ }^{14,16}$. Despite its known role in regulating longevity downstream of the insulin-like signaling ${ }^{15,18}$, the p38-MAPK pathway's role in DR-mediated longevity has not been clearly elucidated.

The present study defines the critical role of the p38-MAPK pathway in ensuring DR-mediated longevity. Using three different paradigms of DR in worms, we show that core components of the p38-MAPK pathway are essential for DR-induced life-span extension. In a genetic model of DR, we show that p38-MAPK pathway does not mediate the DR-induced metabolic shift towards fatty acid oxidation but is required for transcriptional upregulation of the CyTP genes. Further, we show that the levels of polyunsaturated fatty acids (PUFAs), especially linoleic acid (LA) and eicosapentaenoic acid (EPA) increase during DR and that the life span of DR worms is dependent on specific PUFA biosynthesis genes. Importantly, LA and EPA are able to independently activate the CyTP genes, in a p38-MAPK-dependent manner. Overall, our study suggests that the p38-MAPK pathway may respond to products of metabolic reprogramming associated with DR to upregulate CyTP genes. Considering the conserved nature of DR and the p38-MAPK pathway, such coupling may underlie the beneficial role of DR in different dietary paradigms in worms as well as in higher organisms.

\section{Results}

C. elegans p38-MAPK signaling mediates DR-induced life-span extension. In C. elegans, DR may be implemented using genetic mimics as well as by non-genetic interventions. We have shown that RNAi inhibition of a C. elegans ortholog of the human NEK2 (NIMA-related kinase 2) gene, $d r l-1$, mimics a DR-like condition and extends life span, without altering food intake ${ }^{3}$. To identify additional components of the DRL-1 signaling, we screened for the MAPK mutants that failed to show life span extension upon exposure to $d r l-1$ RNAi. We found $d r l-1$ RNAi increased the life span of wild-type (WT) worms (Fig. 1a, also see Supplementary Table 1) but failed to extend the life span of pmk-1(km25) (Fig. 1b), a deletion mutant of the worm p38-MAPK ortholog. The PMK-1 P38-MAPK in C. elegans is activated by the upstream MAPKK, SEK-1 and the MAPKKK, NSY-1. We found that deletion mutants of $n s y-1$ (Fig. 1c) and sek-1 (Fig. 1d) kinases showed no life-span extension upon $d r l-1$ knockdown (KD), confirming the requirement of the p38-MAPK pathway in DRL-1 signaling. We ruled out the possibility of any defect in the RNAi machinery in these mutants by feeding embryonically lethal pos-1 RNAi (Supplementary Fig. 1a); like wild-type, all p38-MAPK pathway mutant larvae failed to reach adulthood on pos-1 RNAi. Also, we observed no changes in the pharyngeal pumping rate between sek-1 and wild-type worms, confirming that inhibition of p38-MAPK signaling does not affect intake of RNAi-producing bacteria (Supplementary Fig. 1b). We further found that this effect on life-span extension of the $d r l-1$ model of DR is specific to p38-MAPK signaling, because the $d r l-1$ RNAi is able to increase life span of $j n k-1(g k 7)$ mutant, component of another prominent MAPK signaling pathway (Supplementary Fig. 1c). Also, inhibition of p38-MAPK signaling is not a limitation for life-span extension as reducing Insulin/IGF-1-like signaling by feeding daf2 RNAi leads to significantly increased life span of the sek-1( $\mathrm{km} 4)$ mutant (Supplementary Fig. 1d).

The eat-2 mutants represent a well-established genetic model of DR in C. elegans ${ }^{19}$. We asked whether the p38-MAPK signaling is also required for the increased life span in this paradigm of DR. We found that sek-1 RNAi significantly suppressed the increased life span of eat-2(ad465), without affecting WT life span (Fig. 2a). Similarly, the life span of eat-2 (ad465);sek-1 ( km4) double mutant is significantly lower than eat2(ad465), suggesting a genetic dependence (Fig. 2b). These results confirm that genetic DR regimes induced by $d r l-1$ RNAi and eat- 2 mutant require a downstream p38-MAPK signaling for life-span extension.

Apart from the genetic models described above, DR in $C$. elegans can also be implemented by serially diluting the bacterial feed (Bacterial Dilution Regime, BDR) ${ }^{20,21}$. Alternatively, feeding glucose analog, 2-deoxyglucose (2-DOG) has been shown to increase life span and mimic DR conditions in worms ${ }^{22}$. We therefore asked whether the p38-MAPK pathway is required for these non-genetic modes of DR. The BDR in WT worms generates a typical bell-shaped curve when average life span is plotted against the bacterial dilutions, showing a consistent increase in life span until an optimum DR threshold is reached. We observed that sek-1(km4) mutants undergoing BDR (Fig. 2c) failed to show any increase in life span when subjected to lower bacterial concentrations, confirming a complete dependence on the p38-MAPK pathway for longevity assurance. In contrast to this, 2-DOG induced DR does not require the p38-MAPK pathway as it was able to increase life span of the sek-1(km4) mutant (Fig. 2d). Together, we show that p38-MAPK is required for life-span extension by certain DR regimes but not others.

p38-MAPK signaling is not required for DR-induced primary metabolic changes. Metabolic changes during DR in C. elegans are generally associated with lowering of fat content, reduction in respiration rate, ROS production, and enhanced autophagy ${ }^{3}$. These changes are essential for increased longevity of the 

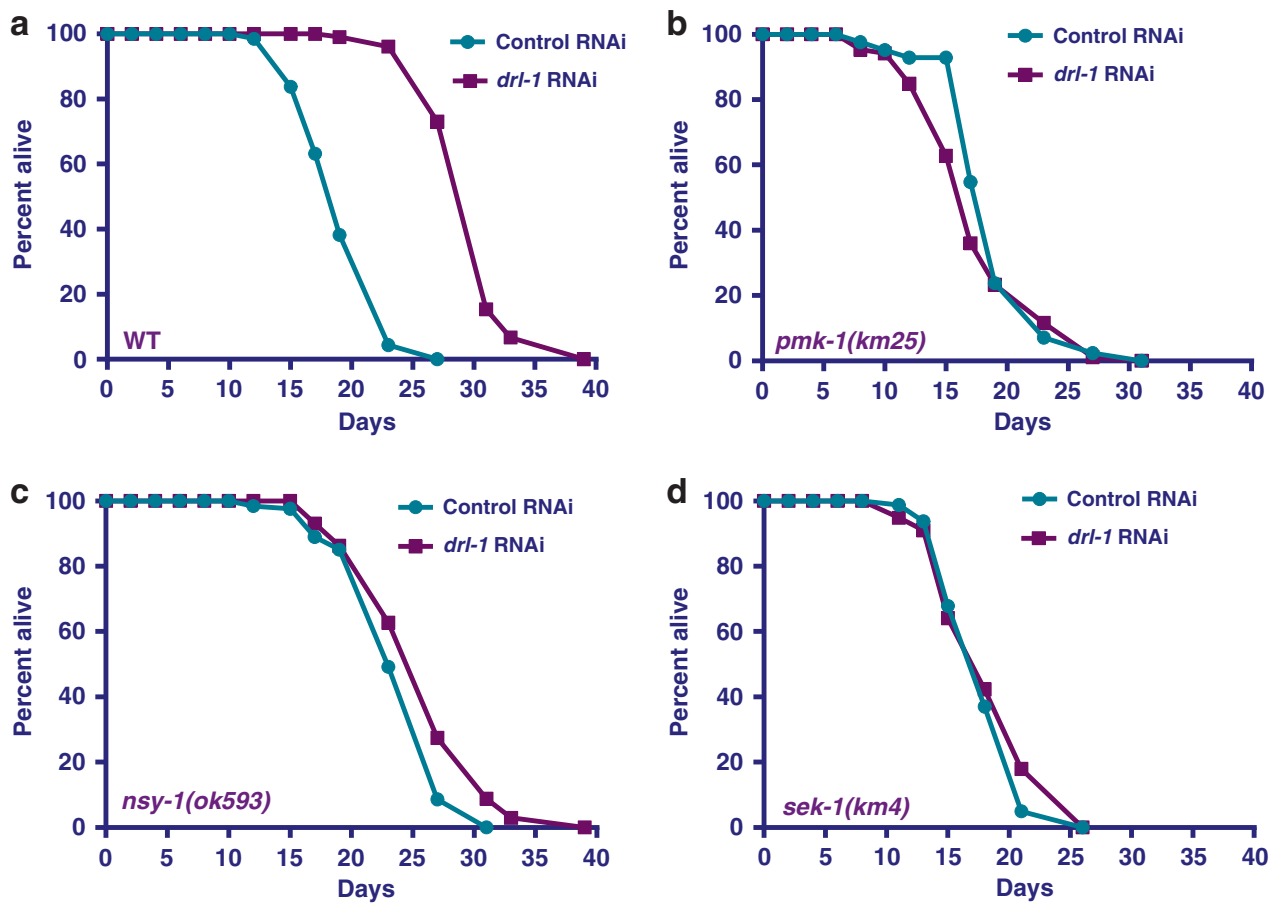

Fig. 1 DR-like condition initiated by $\boldsymbol{d r l} \mathbf{- 1}$ knockdown requires p38-MAPK pathway components. a The life span of wild-type (WT) worms are extended when drl-1 is knocked down using RNAi. b-d The extended life span on drl-1 knockdown is dependent on nsy-1, sek-1, and pmk-1. No life-span extension is observed when drl-1 is knocked down in pmk-1(km25) (b), nsy-1(ok593) (c), or sek-1(km4) (d). Life span was performed at $20^{\circ} \mathrm{C}$ and life-span summary data is provided in Supplementary Table 1.

a
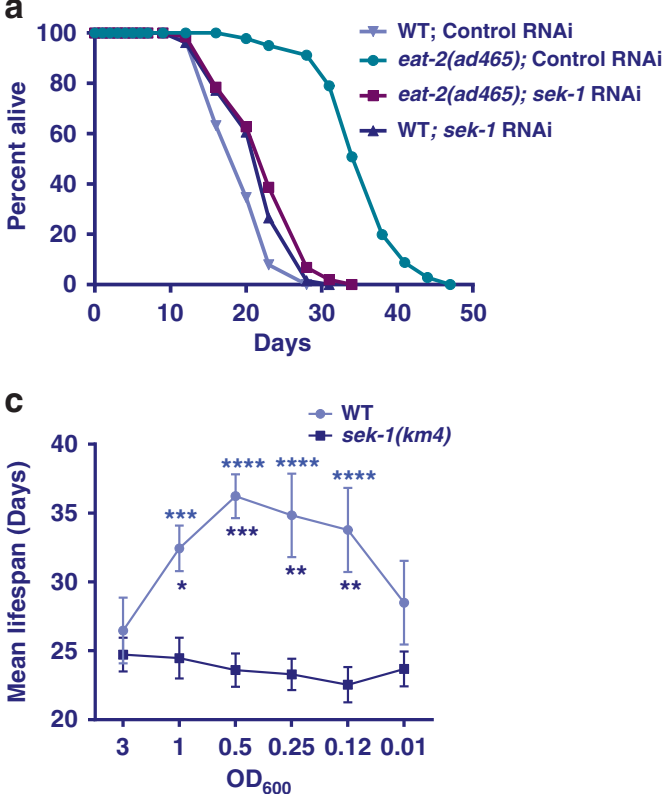

b

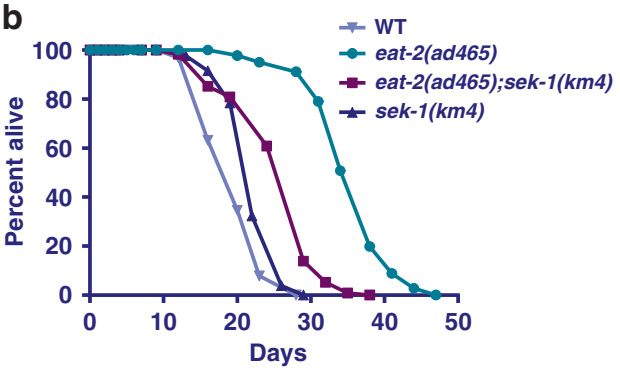

d

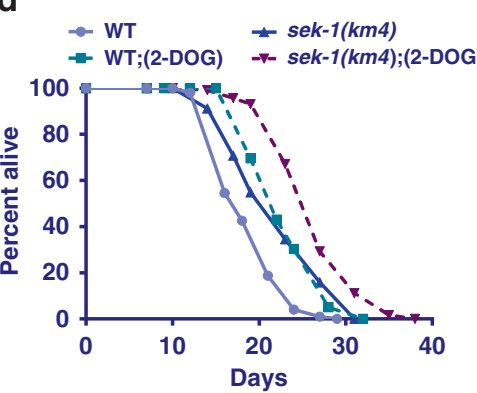

Fig. 2 Other paradigms of DR also require p38-MAPK pathway components. a The life span of eat-2(ad465) is suppressed by sek-1 RNAi. b The increased life span of eat-2(ad465) is partially abrogated in eat-2(ad465);sek-1(km4). The above life spans were performed concurrently. c In bacterial dilutioninduced DR (BDR), decreasing concentration of bacterial food produces a bell-shaped curve in WT. However, in sek-1(km4), no life-span extension is observed at any of the dilutions. Bacterial culture of $\mathrm{OD}_{600}=3.0$ was the starting concentration for serial dilution. The increase in life span when WT was maintained at $\mathrm{OD}_{600}=1.0,0.5,0.25$, or 0.125 was significant as compared to $\mathrm{OD}_{600}=3.0$ (purple stars); the difference in case of sek-1(km4) was not. Two-way ANOVA-Tukey's multiple comparisons test. ${ }^{\star \star \star} P=0.0005,{ }^{\star \star \star \star} P \leq 0.0001$. Also, the average life spans of $W T$ at dilutions $O D_{600}=1.0,0.5$, 0.25 , and 0.125 were significantly different from sek-1 $(\mathrm{km} 4)$ (dark blue stars). Two-way ANOVA-Sidak multiple comparisons test. ${ }^{\star} P=0.0472,{ }^{\star \star} P=$ 0.0015 (for $\mathrm{OD}_{600} 0.25$ ), ${ }^{\star \star} P=0.0021$ (for $\mathrm{OD}_{600} 0.125$ ), ${ }^{\star \star \star} P=0.0005 . n=4$ independent experiments. Data are presented as mean values $+/-\mathrm{SEM}$. d The life span of WT as well as sek-1 $(\mathrm{km} 4)$ is extended to the same extent when the worms were grown on 2-deoxyglucose (2-DOG). Life span was performed at $20^{\circ} \mathrm{C}$ and life-span summary data is provided in Supplementary Table 1. 
a

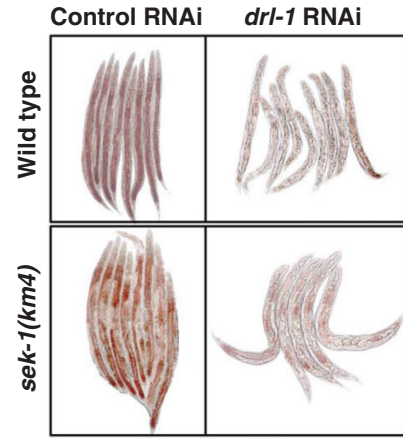

C
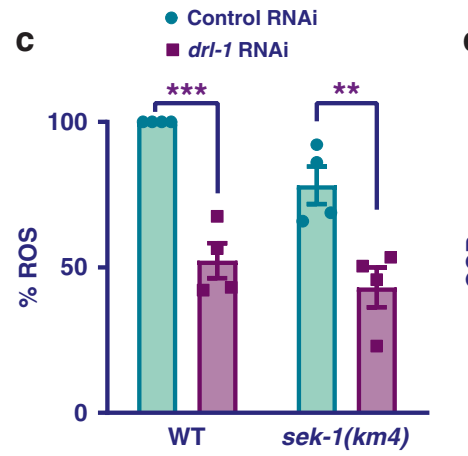

b

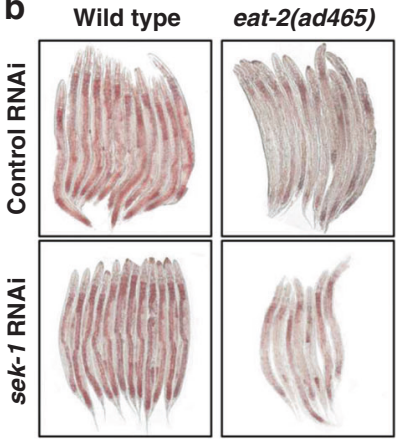

d

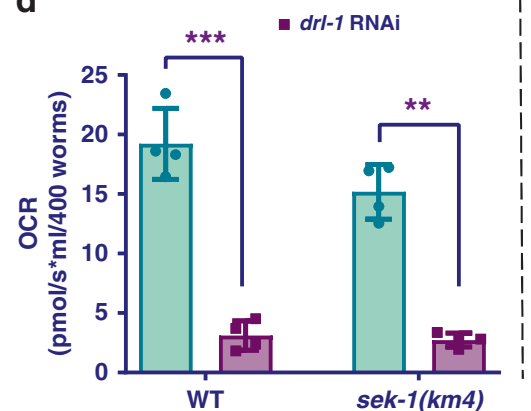

e

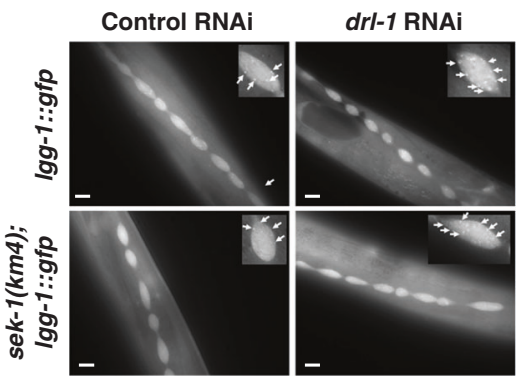

- Control RNAi - drl-1 RNAi

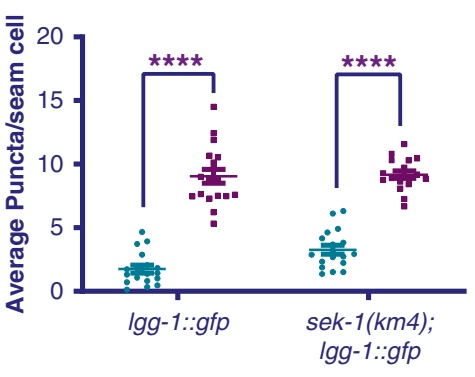

Fig. 3 Metabolic reprogramming associated with DR does not require p38-MAPK. a Knocking down drl-1 produces a DR-like phenotype in both WT as well as sek-1(km4), resulting in depletion of stored fat to similar extent. Quantified data is provided in Supplementary Fig. 2a. One of three independent experiments is shown. b Depletion of stored fat during DR, as seen in eat-2(ad465), is independent of sek-1. The sek-1 RNAi did not significantly change the low fat of eat-2(ad465). Quantified data is provided in Supplementary Fig. 2b. One of three independent experiments is shown. Multiple overlapping images were acquired at $\times 100$ magnification to cover the entire worm body and stitched together to generate a contiguous image. $\mathbf{c}$ Knockdown of $d r l-1$ decreased ROS levels to similar extent in WT and sek-1(km4), as determined by DCFDA assay. Data are presented as mean values \pm SEM. $n=4$ independent experiments. Two-way ANOVA-Sidak's multiple comparisons test, ${ }^{\star \star \star} P=0.0004,{ }^{\star \star} P=0.0050$. d Oxygen consumption rate (OCR) measured using Oroboros O2K shows similar respiratory rates in WT and sek-1 $(\mathrm{km} 4)$. Data are presented as mean values \pm SEM. $n=4$ independent experiments. Unpaired two-tailed $t$ test, ${ }^{\star \star} P=0.0011,{ }^{\star \star \star} P=0.0006$. e Autophagy, as determined by puncta formation in the seam cells of a LGG-1::GFP-expressing strain (upper panel), was increased in both WT as well as in sek-1 $(\mathrm{km} 4)$ when $\mathrm{drl}-1$ was knocked down by RNAi. Representative images (upper panel) and quantification (lower panel) from one of two independent experiments shown. Experiments performed at $20^{\circ} \mathrm{C} . n=18$ worms. Data are presented as mean values \pm SEM. Two-way ANOVA-Sidak's multiple comparisons test, ${ }^{\star \star \star \star} P \leq 0.0001$. Scale bar $=10 \mu \mathrm{m}$. Experiments were performed at $20^{\circ} \mathrm{C}$. Source data are provided as a Source data file.

organism. Hence, we asked if any of these important cellular changes are mediated by the p38-MAPK signaling. We tested fat content of $d r l-1$ RNAi-fed wild-type and sek-1( $\mathrm{km} 4)$ worms after staining them with Oil Red O stain and found that the fat content was significantly decreased for both strains (Fig. 3a, Supplementary Fig. 2a, related to Fig. 3a). Similarly, we also observed that there was no change in the fat content of eat-2(ad465) when p38-MAPK signaling was inhibited by either feeding sek-1 RNAi (Fig. 3b, Supplementary Fig. 2b, related to Fig. 3b) or on crossing with sek-1(km4) (Supplementary Fig. 2c). These observations suggest that p38-MAPK signaling is not required for DR-induced changes in fat reserves. We also quantified the ROS levels in $d r l-1$ RNAi-treated wild-type and sek-1(km4) mutant worms by DCFDA assay and found similar decreases in both strains (Fig. 3c). In addition, $d r l-1$ knockdown worms exhibited lower respiratory rate (Fig. 3d) and higher autophagy that were not dependent on the p38-MAPK pathway (Fig. 3e). We also found that the eat-2(ad465) worms have similar trends in respiratory rate (Supplementary Fig. 3a) and autophagy (Supplementary Fig. 3b). All together, these results show that p38-MAPK signaling is not required for primary metabolic changes induced by genetic DR regimes.

Transcriptional upregulation of CyTP genes during DR-like state requires p38-MAPK signaling. We have earlier reported that metabolic reprogramming during DR leads to increased expression of CyTP XDP genes ${ }^{3}$, possibly to detoxify lipophilic endotoxins generated during increased fatty acid oxidation or to catalyze reactions to generate PUFA derivatives that are important signaling intermediates. Since the above results show that metabolic reprogramming during DR does not require p38MAPK signaling, we next asked whether it mediates transcriptional upregulation of CyTP genes. For this, we compared the mRNA levels of a few selected, annotated Phase-I and Phase-II detoxification genes in wild-type and sek-1(km4) worms grown on control or $d r l-1$ RNAi. We found that expression of these genes increased on $d r l-1 \mathrm{RNAi}$, as reported earlier ${ }^{3}$. Interestingly, in sek-1 $(\mathrm{km} 4)$ the expression of several of these genes is deregulated and $d r l-1$ knockdown could not increase their expression to the same extent as that in wild-type (Fig. 4a). We also validated the sek-1 dependence using a transcriptional GFP reporter of the cyp-35B1 gene (Pcyp-35B1::gfp) (Fig. 4b). The expression of GFP increased when worms were exposed to $d r l-1$ RNAi; however, expression was suppressed upon simultaneous introduction of sek-1 RNAi. We confirmed that the sek-1 RNAi led to dramatic decrease in the levels of activated p38-MAPK/PMK-1, without affecting its levels (Supplementary Fig. 4). These results suggest that p38-MAPK pathway may regulate DR-induced longevity by inducing CyTP genes, in response to products of the reprogrammed metabolism (e.g., fatty acids or their derivatives). When 
a

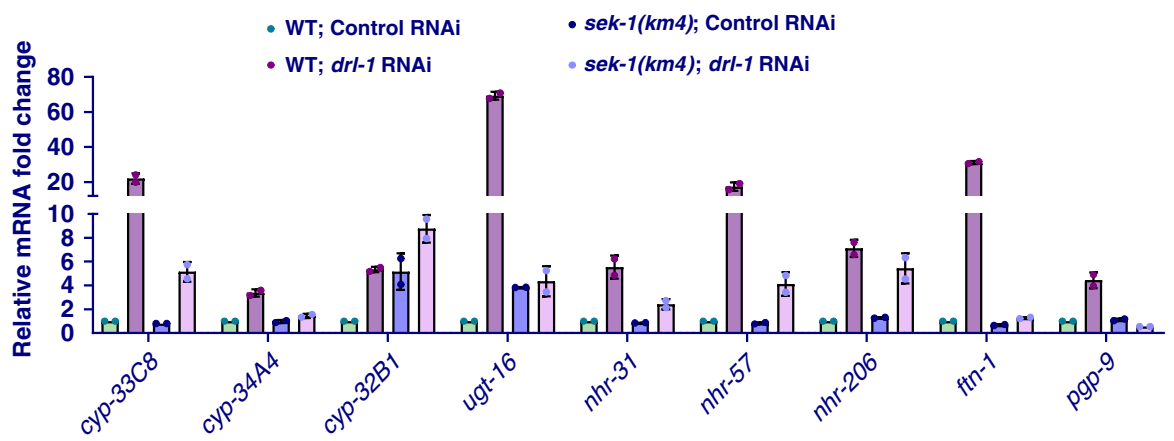

b
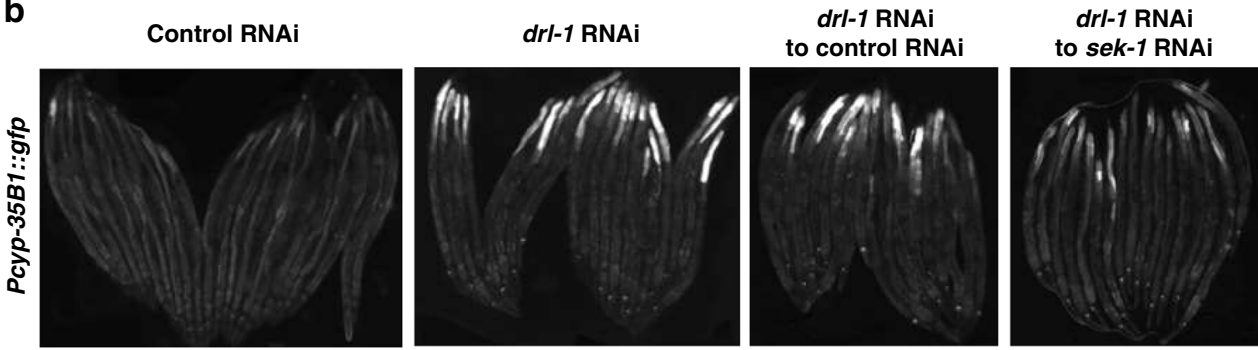

Fig. 4 CyTP genes are upregulated during DR-like condition in a p38-MAPK-dependent manner. a Quantitative reverse transcriptase PCR (QRT-PCR) analysis shows that many Cytoprotective genes (CyTP) genes, of the GO category "xenobiotic detoxification pathway" (XDP), that are upregulated when $\mathrm{drl}-1$ is knocked down in WT, but failed to do so to the same extent in sek-1 $(\mathrm{km} 4)$. Expression levels are normalized to actin. $n=2$ biologically independent samples. Data are presented as mean values \pm std. dev. b The expression of Pcyp-35B1::gfp is enhanced at YA stage when drl-1 is knocked down using RNAi, compared to control RNAi. However, the upregulation is abrogated if sek- 1 is also knocked down by RNAi. Representative images from one of three independent experiments shown. Multiple overlapping images were acquired at $\times 100$ magnification to cover the entire worm body and stitched together to generate a contiguous image. Experiments performed at $20^{\circ} \mathrm{C}$. Source data are provided as a Source data file.

the pathway is abrogated, the expression of these genes is deregulated and they become recalcitrant to context-specific activation during DR.

PUFAs generated during genetic DR regimes upregulate CyTP through p38-MAPK signaling. Since the $d r l-1 \mathrm{KD}$-induced CyTP gene upregulation is dependent on NHR- $49^{3}$ as well as p38-MAPK signaling (this study), we explored the role of PUFAs in this process. The reason behind this is NHR-49, which apart from regulating the fatty acid oxidation genes, also modulates expression of genes encoding the $\Delta-9$ desaturase enzymes FAT-5, FAT-6, and FAT- $7^{23}$. These enzymes are essential for biosynthesis of PUFAs (Fig. 5a). In addition, previously published reports have found that the levels of PUFA increase during nutrient stress $^{24}$ and various PUFAs have been shown to be important for maintaining the activity of the p38-MAPK ${ }^{25}$. We, therefore, hypothesized that the enhanced transcription of CyTP genes via the p38-MAPK pathway during DR may be due to the increased levels of PUFAs. We investigated this hypothesis by genetic and biochemical approaches using mutants defective in PUFA synthesis and GC-MS analysis to directly measure levels of PUFAs, respectively. We first performed $d r l-1$ RNAi life-span analysis in mutants defective in PUFA biosynthesis. We used fat6; fat-7 double mutant and fat-2 mutant lacking $\Delta 9$ - and $\Delta 12$ desaturase activities, respectively; consequently, these mutants are deficient in downstream PUFA biosynthesis ${ }^{26-28}$ but may accumulate monounsaturated oleic acid (OA) or saturated stearic acid (SA) and palmitic acid (PA) (Fig. 5a). We found that $d r l-1$ RNAi failed to increase life span in these mutants (Fig. 5b, c) highlighting an essential role of PUFAs in $d r l-1 \mathrm{KD}$-induced DR longevity. Similarly, in the eat-2(ad465), knocking out fat-2 led to suppression of life span (Fig. $5 \mathrm{~d}$ ). We also performed BDR assay with fat-2(wa17) and found that in the mutant, the mean life spans, when plotted against bacterial dilutions, failed to produce a bell-shaped curve, typically seen in WT (Fig. 5e). These results show that when PUFA biosynthesis is perturbed, genetic as well as non-genetic DR regimes fail to increase life span, suggesting an involvement of the downstream PUFAs or their derivatives in activating p38-MAPK during DR.

We next asked if the failure to increase life span following $d r l-1$ $\mathrm{KD}$ in the PUFA-defective mutants is possibly due to abrogated transcriptional regulation of CyTP genes. For that, we grew the fat-2 mutant on control or $d r l-1$ RNAi and determined the mRNA expression of the CyTP genes (Fig. 5f). Interestingly, we found that the mRNA expression of several of the CyTP genes was already upregulated (significantly for $f t n-1, c y p-35 B 1$, cyp$32 B 1$, gst-19, and $n h r-206)$ in the fat-2 mutant. This deregulation of CyTP genes is accompanied by the enhanced p38-MAPK activity, as determined by a phospho-PMK-1 (pPMK-1) western blot analysis (Fig. 5g). However, unlike in wild-type, drl-1 RNAi failed to produce similar levels of upregulation in many of these CyTP genes in the fat-2 mutants (Fig. 5f). Consistent with the observation associated with CyTP genes expression, $d r l-1$ RNAi also failed to further increase the p38-MAPK activity in fat-2 mutants (Fig. 5g). The fat-2(wa17);Pcyp-35B1::gfp worms also showed increased expression compared to wild-type; $d r l-1$ RNAi failed to increase the $g f p$ expression beyond this level (Fig. 5h).

Since p38-MAPK is ectopically activated in the fat-2 mutant that blocks PUFA biosynthesis, it suggests that OA, SA or PA may also independently activate the CyTP genes, in a p38MAPK-dependent manner. Indeed, we observed that OA, SA, and PA activated p38-MAPK as well as enhanced the expression of XDP reporter gene Pcyp-35B1::gfp, dependent on a functional p38-MAPK pathway (Supplementary Fig. 5a, b). These results suggest that p38-MAPK as well as the CyTP genes may be ectopically activated in fat-2 mutant worms due to the 
a
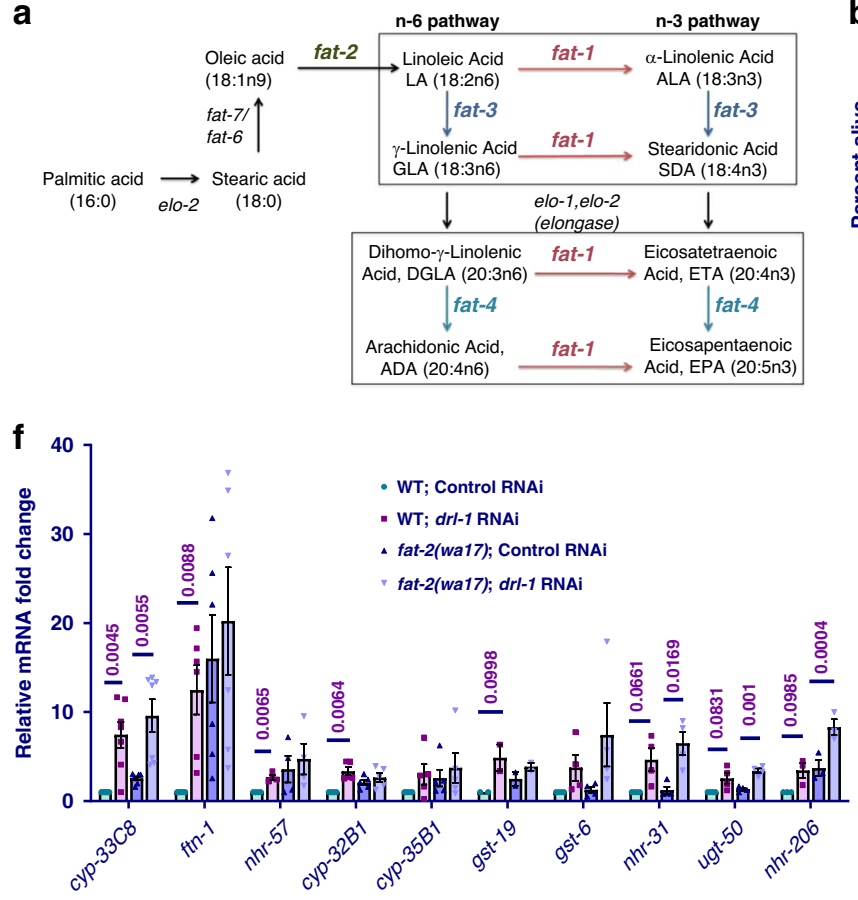

b

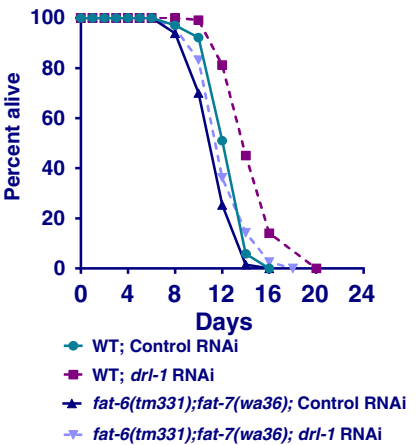

d

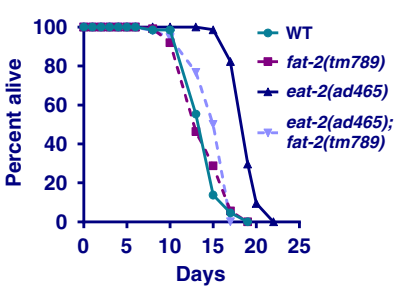

C
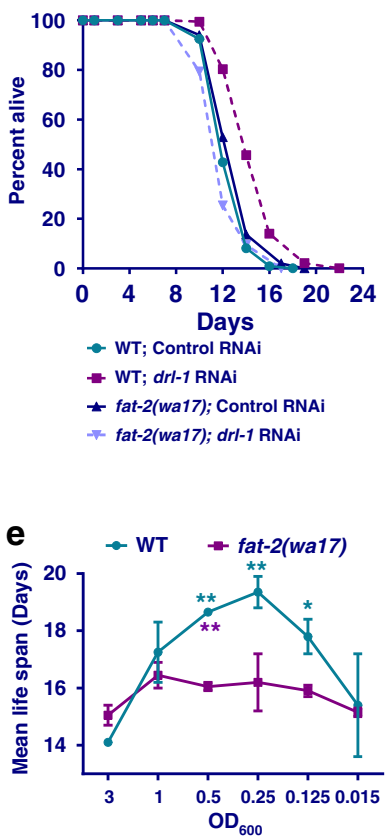

g

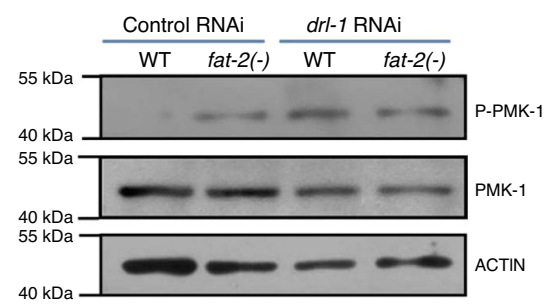

h Control RNAi

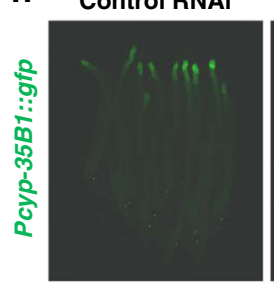

drl-1 RNAi

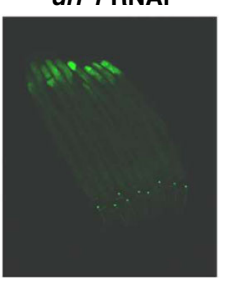

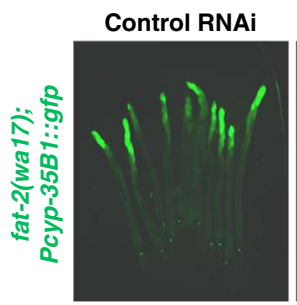

drl-1 RNAi

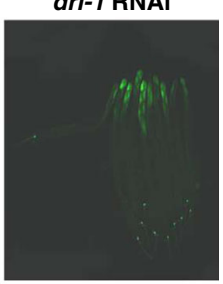

Fig. 5 PUFA biosynthesis genes are required for drl-1-mediated life-span extension. a Schematic representation of the PUFA biosynthesis pathway in $C$. elegans. b, c Life span is extended when $d r l-1$ is knocked down in WT, but not to the same extent in fat-6(tm331);fat-7(wa36) (b) or fat-2(wa17) (c). d Life span of eat-2(ad465) is suppressed in eat-2(ad465);fat-2(tm789). e In bacterial dilution-induced DR (BDR), fat-2(wa17) failed to produce a bell-shaped curve like WT. Bacterial culture of $\mathrm{OD}_{600}=3.0$ was the starting concentration for serial dilution. The increase in life span when WT was maintained at $\mathrm{OD}_{600}=0.5,0.25$, or 0.125 was significant as compared to $\mathrm{OD}_{600}=3.0$ (green stars); the difference in case of fat-2(wa17) was not. Two-way ANOVATukey's multiple comparisons test. ${ }^{\star} P=0.0346,{ }^{\star \star} P=0.0087$ (for $\mathrm{OD}_{600} 0.5$ ), ${ }^{\star \star} P=0.0029$ (for $\mathrm{OD}_{600} 0.25$ ). The average life spans of WT at dilutions $\mathrm{OD}_{600}=0.5$ was significantly different from fat-2(wa17) (purple stars). Unpaired two-tailed $t$ test. ${ }^{\star \star} P=0.0036$. Life spans were performed at $25^{\circ} \mathrm{C}$. Data presented as mean life span \pm SEM. $n=2$ biologically independent replicates. Life-span summary is provided in Supplementary Table 1. $\mathbf{f}$ The expression of selected CyTP genes are upregulated when $d r l-1$ is knocked down. The expression of several of them are dependent on fat-2, as determined by QRT-PCR. Expression levels were normalized to actin. $n=7$ (cyp-33C8), $n=6$ (ftn-1), $n=5$ (cyp-32B1, cyp-35B1), $n=4$ (gst-6, nhr-31, ugt-50), n=3 (nhr-206), or $n=2$ (gst-19) biologically independent samples. Data are presented as mean values \pm SEM. Unpaired two-tailed $t$ test. $\mathbf{g}$ Western blot analysis of WT and fat-2 (wa17) showing that $d r-1$ RNAi upregulates phospho-PMK-1. The levels of phospho-PMK-1 is already upregulated in fat-2(-) and is not further upregulated when $d r l-1$ is knocked down. One of two independent experiments shown. $\mathbf{h}$ The expression of Pcyp-35B1::gfp is enhanced at YA stage when drl-1 is knocked down using RNAi, compared to control RNAi. However, fat-2(wa17);Pcyp-35B1::gfp already has elevated expression of GFP, and drl-1 RNAi failed to further increase it. Representative images from one of three independent experiments shown. Multiple overlapping images were acquired at $\times 100$ magnification to cover the entire worm body and stitched together to generate a contiguous image. Source data are provided as a Source data file. All experiments were performed at $25^{\circ} \mathrm{C}$.

accumulation of upstream fatty acids or their derivatives. As a result, they become recalcitrant to context-specific activation during DR and life-span benefits are suppressed. Altogether, these results highlight the important role of a balance between SFA, MUFA, and PUFA in regulation of CyTP genes downstream of the p38-MAPK pathway to regulate longevity during DR.

Further, to identify specific PUFA molecules that may be signaling to the $\mathrm{p} 38$-MAPK pathway under a DR-like condition, we quantified PUFAs in worms subjected to drl-1 RNAi using GC-MS. We found that levels of several SFAs and MUFAs like C14:0, C15:0iso, C16:0, C16:1, and C17: $\Delta$ were significantly lower in $d r l-1$ RNAi worms compared to control RNAi (Fig. 6a). These fatty acids are abundant components of $C$. elegans triglyceride (TAGs) ${ }^{29}$. Lower levels of these fatty acids are consistent with our finding that $d r l-1$ RNAi results in lower fat stores in worms. In contrast, we found that the levels of PUFAs, including C18:2, C20:4, C20:4(n3), and C20:5 are significantly higher in $d r l-1$ RNAi-treated wild-type worms, supporting the important role of PUFAs during DR (Fig. 6a). As expected, we did not observe any major differences between the fatty acid profile of sek-1 and wildtype worms treated with $d r l-1$ RNAi (Fig. 6a), strengthening our conclusion that p38-MAPK signaling may act downstream of 


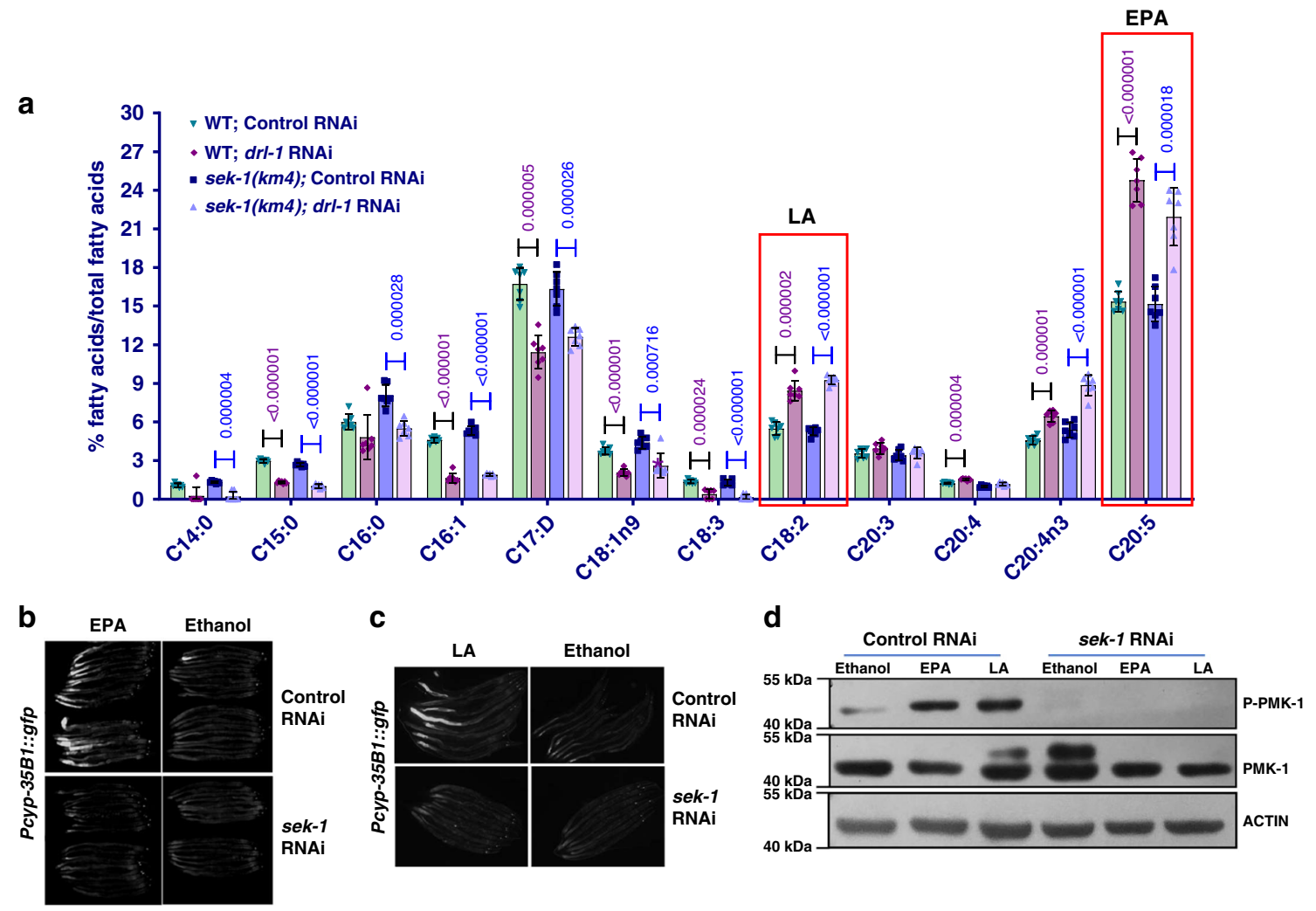

Fig. 6 Eicosapentaenoic acid (EPA) and linoleic acid (LA) supplementation activates p38-MAPK. a GC-MS analysis revealed that PUFAs are differentially regulated when drl-1 is knocked down in WT. The levels of these PUFAs change in a similar manner when drl-1 is knocked down in sek-1(km4). $n=7$ biologically independent samples. Data are presented as mean values +/- std. dev. Unpaired two-tailed $t$ test. b, c The Pcyp-35B1::gfp worms were grown on control or sek-1 RNAi plates supplemented with EPA (b) or LA (c) since hatching. PUFA supplementation increased the expression of Pcyp-35B1: gfp when worms are grown on control RNAi but not on sek-1 RNAi. Multiple overlapping images were acquired at 100X magnification to cover the entire worm body and stitched together to generate a contiguous image. One of two (LA) or three (EPA) independent experiments shown. $\mathbf{d}$ Western blot analysis of Pcyp-35B1::gfp grown on control or sek-1 RNAi showing that EPA and LA supplementation upregulates phospho-PMK-1 levels, in a sek-1dependent manner. One of three independent experiment shown. Source data are provided as a Source data file. All experiments were performed at $20{ }^{\circ} \mathrm{C}$.

fatty acid metabolism. We also performed GC-MS analysis of the eat-2(ad465) and compared it to that of wild-type (Supplementary Fig. 6) and found similar trend in most cases except for 18:2 fatty acid which may point to DR paradigm-specific differences.

Among the PUFAs detected in $d r l-1$ RNAi worms, levels of linoleic acid (C18:2n6; LA) and eicosapentaenoic acid (20:5n3; EPA) were significantly upregulated in wild-type grown on $d r l-1$ RNAi. So, we asked if these specific PUFAs could independently activate CyTP genes in a p38-MAPK signaling-dependent manner, bypassing the requirement of DR for metabolic reprogramming. We supplemented the reporter Pcyp-35B1::gfp worms with EPA or LA and found that the GFP expression was enhanced (Fig. 6b, c). Most importantly, this upregulation was dependent on p38-MAPK signaling as the increase was abrogated when sek-1 was KD using RNAi (Fig. 6b, c). Further, supplementing EPA or LA were sufficient to activate PMK-1 as determined by phospho-western analysis (Fig. 6d), independent of $d r l-1$ signaling. Together, these experiments show a critical role of specific PUFAs in inducing CyTP genes via p38-MAPK pathway during DR.

Next, we asked whether these specific PUFAs, LA and EPA, are sufficient to mediate the signaling events that results in increased life span of worms undergoing genetic DR. We grew wild-type or PUFA-defective fat-2(wa17) mutant on control or drl-1 RNAi, in the presence or absence of LA or EPA and scored life span. The life span of WT was slightly increased by LA supplementation; but EPA decreased wild-type life span (Fig. 7a). However, in fat-2 (wa17) mutant background, both LA and EPA increased life span. Importantly, while in fat-2(wa17) mutant, where $d r l-1 \mathrm{KD}$ failed to increase life span, life span was increased when the mutant was supplemented with LA or EPA (Fig. 7b).

Together, these experiments show that PUFA or its derivatives produced during DR activates p38-MAPK pathway and CyTP gene expression to mediate longevity benefits.

\section{Discussion}

A metabolic shift to fatty acid utilization has been shown to play a prominent role in DR-mediated life-span extension in multiple model systems $2,6,30$. However, apart from supporting efficient energy utilization, we still lack a complete mechanistic understanding of the multitude of potential benefits that this shift endows. We have earlier shown that metabolic reprogramming to fatty acid oxidation during DR in C. elegans leads to a coupled activation of the cytoprotective genes, members of which are categorized as XDP genes according to Gene Ontology terms. This coupling is a requirement of DR-mediated life span extension in the two genetic DR models tested ${ }^{3}$. However, how the organism transmits the information of metabolic reprogramming to activate gene expression during DR was not known. In the present study, we show that PUFAs, especially LA and EPA, or possibly derivatives of it, which accumulate during DR, can act as potential signaling molecules to upregulate CyTP genes via the conserved p38-MAPK pathway (Fig. 7c). Previously, DR has been independently shown to upregulate $\mathrm{EPA}^{31}$ as well as activate XDP 


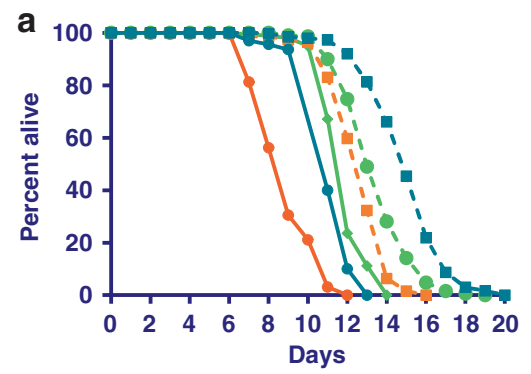

$$
\begin{aligned}
& \rightarrow \text { WT (Control RNAi); Ethanol } \\
& - \text { WT (drl-1 RNAi); Ethanol } \\
& - \text { WT (Control RNAi); EPA } \\
& - \text { WT (drl-1 RNAi); EPA } \\
& \approx \text { WT (Control RNAi); LA } \\
& - \text { WT (drl-1 RNAi); LA }
\end{aligned}
$$

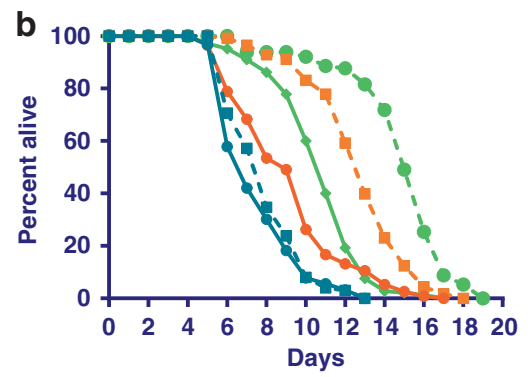

$$
\begin{aligned}
& \text { - fat-2(wa17) (Control RNAi); Ethanol } \\
& - \text { fat-2(wa17) (drl-1 RNAi); Ethanol } \\
& \text { - fat-2(wa17) (Control RNAi); EPA } \\
& \text { - fat-2(wa17) (drl-1 RNAi); EPA } \\
& \approx \text { fat-2(wa17) (Control RNAi); LA } \\
& - \text { fat-2(wa17) (drl-1 RNAi); LA }
\end{aligned}
$$

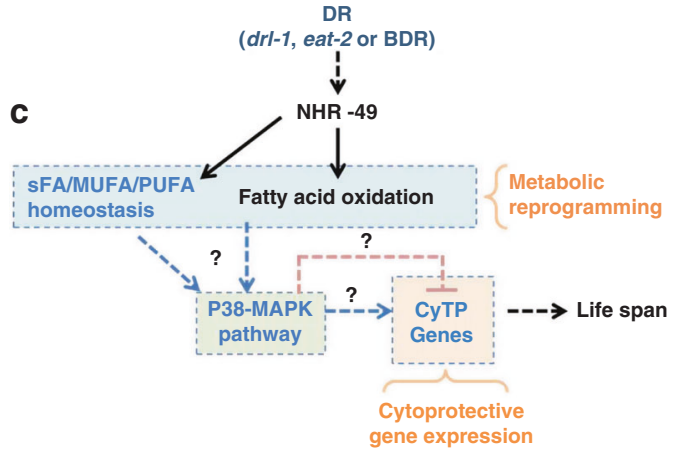

Fig. 7 External supplementation of EPA or LA rescues life-span defects of fat-2(wa17). a, b WT (a) or fat-2(wa17) (b) were grown on control or drl-1 RNAi in the presence or absence of LA or EPA. In fat-2(wa17), the drl-1 RNAi failed to increase life span. However, the life span was rescued on addition of LA or EPA. Life span was performed at $25^{\circ} \mathrm{C}$ and life-span summary data is provided in Supplementary Table 1. c A model showing that p38-MAPK functions downstream of different paradigms of DR to regulate gene expression and longevity, as discovered in this study. The '?' denotes unanswered questions that evolve from the study. For example, How does sFA/MUFA/PUFAs or their derivatives activate p38-MAPK? What transcription factors function downstream of p38-MAPK to regulate CyTP genes? Source data are provided as a Source data file.

genes $^{32,33}$ in mammals. Our study, using C. elegans, describes a previously unknown role of the PUFA-p38-MAPK signaling axis in cyto-protection and DR-mediated longevity assurance. Although, we have mostly used a genetic model of DR in our study, conservation of the components suggest that similar mechanisms may drive pro-longevity effects in other DR paradigms in C. elegans as well as in other species.

In C. elegans, metabolic reprogramming during nutrient deprivation is under the transcriptional control of NHR- 49 that regulates the expression of both beta-oxidation as well as PUFA biosynthetic genes 23,34 . The lipid molecules may act as a doubleedged sword. While metabolic shift to fatty acid oxidation in worms leads to efficient energy utilization during DR as well as reduced ROS production ${ }^{3,35,36}$, an imbalance may be detrimental to the cells. Lipid molecules in the cellular milieu are prone to oxidative modifications that are hazardous. PUFA molecules are extremely prone to peroxidation, whereby free radicals attack carbon-carbon double bonds in the lipids $s^{37,38}$. PUFA peroxides such as malondialdehydes and 4-hydroxynonenal (4-HNE), are the most extensively-studied lipid peroxidation end-product and are damaging to cells. Interestingly, 4-HNE shows physiological and protective function as a signaling molecule that stimulates gene expression. However, it also shows cytotoxicity by inhibiting gene expression and promoting the development and progression of different pathological states ${ }^{39}$. Oxidized omega-3 PUFA has been shown to induce oxidative stress and inflammation ${ }^{40}$. In contrast, deuterated PUFAs retard lipid peroxidation and is capable of regulating oxidative stress, aging, and providing life-span benefits in C. elegans ${ }^{41}$. In addition, PUFAs are associated with lipid membranes from where they are released by phospholipase A2 and undergo processing to many biologically active signaling molecules in mammals like prostaglandins (PGs), prostacyclins and thromboxanes (TXs), lipoxins (LXs) and leukotrienes (LTs), protectins, marensins, and resolvins, epoxyeicosatrienoic acids (EETs), and hydroxyeicosatetraenoic acids (HETEs) that have pro- and anti-apoptotic properties, play important roles in inflammation, modulate the immune responses, and other unknown processes ${ }^{42}$. Although C. elegans lack cyclooxygenase orthologs, several F-series prostaglandins are synthesized, using a cyclooxygenase-independent pathway, from C20 PUFA precursors in the female germline that help to attract sperm to oocytes ${ }^{29}$. Thus, an elevated level of various PUFAs (like LA or EPA) as observed on drl-1 RNAi and in the eat-2 mutant may result in increased peroxidation that are potentially detrimental to the organism, or can lead to dysregulation of key signaling events, if not properly contained by detoxification. These may be perceived as danger or stress signals that could activate the cytoprotective processes through p38-MAPK pathway. The xenobiotic detoxification system is capable of detoxifying a plethora of lipotoxins including steroids, eicosanoids, and other fatty acid derivatives that may constitute the lipophilic endotoxins that are byproducts of increased fatty acid metabolism. DR has indeed been shown to activate the phase-II detoxification system both at transcriptomic as well as biochemical level in mammals ${ }^{32,33}$. It may, however, be noted that while many of these cytoprotective genes are classified as XDP genes according to Gene Ontology 
analysis, they have other significant functions. For example, CYP22A1 or DAF-9 produces a bile acid-like hormone that binds to DAF-12 receptor to control development and longevity ${ }^{43-45}$. Others have important function in fat storage regulation ${ }^{46}$ or directional sperm motility ${ }^{47}$. At the biochemical level, CYP-29A3 and CYP-33E2 are the major CYP isoforms that have been shown to be involved in EPA metabolism ${ }^{48}$ and may have important signaling roles. Together, they may contribute to cyto-protection and signaling required for enhanced longevity. So, there is merit in coupling metabolic reprogramming to activation of these cytoprotective machineries during DR through the stressresponsive $\mathrm{p} 38$-MAPK pathway.

We have shown here that DR or external supplementation of LA and EPA induce the p38-MAPK pathway, both in terms of increased PPMK-1 levels as well as increased CyTP gene expression. Previous studies have also shown that p38-MAPK pathway may be activated by PUFAs and other fatty acids under different physiological conditions. External supplementation experiments have shown that PUFAs have beneficial effects on life span and innate immunity by activating the p38-MAPK pathway in C. elegans ${ }^{49}$. The fat-3 mutants defective in $\gamma$-linolenic acid (GLA) and stearidonic acid (SDA) biosynthesis have reduced basal activity of p38-MAPK that can be rescued by external supplementation of these lipids ${ }^{25}$. Arachidonic acid activates members of the mitogen-activated protein kinase superfamily in rabbit proximal tubule cells ${ }^{4}$, hepatocytes ${ }^{50}$, and human brain endothelial cells (HBECs) ${ }^{51}$. The MUFA oleic acid has been shown to activate p38-MAPK in hepatocytes ${ }^{52}$ and in rat hepatoma dRLh-84 cells ${ }^{53}$. In vascular endothelial cells, LA activates p38-MAPK within $10 \mathrm{~min}$ of treatment ${ }^{54}$. In primary hepatocytes, fasting-induced free FAs activate $\mathrm{p} 38$-MAPK providing a likely reason of this pathway acting as a metabolic sensor for increased PUFA and associated threat during $\mathrm{DR}^{55}$. Our current and previous studies, in combination, provides a similar context for activation of p38-MAPK and CyTP genes to counter stress, potentially generated by lipophilic endotoxins produced due to metabolic reprogramming.

However, the question remains about the mechanisms of activation of the p38-MAPK pathway by fatty acids during DR. Levels of omega-3 PUFAs have been shown to enhance sensitivity of TRPV channels by altering membrane fluidity and modulating $\mathrm{Ca}^{2+}$ levels ${ }^{56}$. Also, in fat-3 mutants, diminished calcium response and associated avoidance response is rescued by 20 carbon omega- 6 and omega- 3 PUFAs ${ }^{57}$. In addition, TRP-like channels mediate upregulation of $\alpha$-dicarbonyl detoxification system in a p38-MAPK-dependent manner ${ }^{58}$. Thus, it is tempting to speculate that increase in PUFA levels during DR maybe activating p38-MAPK pathway and associated CyTP genes by increasing sensitivity of TRP channels. PUFAs can also activate p38-MAPK through the generation of ROS, and supplementation of Vitamin $\mathrm{E}$ is able to abrogate this response $\mathrm{e}^{59}$. However, activation through ROS seems counter intuitive, because DR reduces the level of these molecules. Further investigation is required to decipher the mechanisms of p38-MAPK activation by PUFAs during DR.

A delicate balance of SFA/MUFA/PUFA levels are maintained within the cell for optimal function of the structural and signaling elements. The toxic effects of PUFA imbalance is particularly apparent in the germline. Dietary supplementation of omega-6 PUFA-dihomogamma linolenic acid (DGLA, 30:3n6) results in sterility due to destruction of germ cells ${ }^{60,61}$. Strains that have higher detoxification capabilities, like the Insulin/IGF1-like signaling receptor daf-2 mutant worms are able to resist DGLA-induced germline sterility ${ }^{61}$. Since PUFAs are more likely to undergo peroxidation, which in turn increases ROS production, long-lived worms like daf-2 mutants have increased
MUFA with decreased PUFA levels ${ }^{62}$. External supplementation of MUFAs and PUFAs have shown opposite effects with the former suppressing and later sensitizing the cells to lipid peroxidation $^{38}$. Dietary supplementation with fish oil increased levels of lipid peroxidation and induced shorter life span in $C$. elegans $^{63}$. On the other hand, lower concentrations of omega- 6 PUFAs activate autophagy and increase life $\operatorname{span}^{24}$. Since PUFAs may be rapidly turned over, it is possible that oxidized PUFAs are promptly eliminated from the system and worms that have active detoxification/elimination machinery may actually benefit even from increased PUFA levels, as seen in this study. The careful balancing of PUFA metabolism is also apparent in mammals where neural stem cell niche triglycerides are enriched in oleic acids and infusing oleic acid directly into brain ventricles is sufficient to inhibit neural stem cell proliferation ${ }^{64}$. Our GC-MS data shows that both $d r l-1 \mathrm{KD}$ and eat-2 mutant worms are low in saturated fatty acids and MUFAs, while some PUFA levels are elevated, providing life-span benefits. However, a disbalance caused by fat-2 mutation leads to deregulation of the p38-MAPK pathway, its associated gene expression as well as suppression of DR-mediated life span.

The C. elegans p38-MAPK pathway is involved in diverse aspects of cellular function, including development ${ }^{65,66}$, stress response ${ }^{14,18}$, innate immunity ${ }^{15,25,67,68}$, metabolism, and longevity 69,70 . In all these cases, when a component of the p38MAPK pathway was mutated, the phenotype was adversely affected. For example, the worms deficient in p38-MAPK signaling were found to be susceptible to Pseudomonas aeruginosa infection ${ }^{15,68}$ and decline in p38-MAPK signaling was shown to cause immune senescence ${ }^{67}$. The $\mathrm{p} 38$-MAPK pathway mutants were susceptible to oxidative stress ${ }^{14}$. The increased life span of daf- 2 mutants ${ }^{15}$ and the food-type dependent life span of $f l r-4$ mutant are dependent on p38-MAPK pathway components ${ }^{70}$. In most of the cases, the activation of the p38-MAPK pathway correlates with the phenotype. For instance, the phosphorylation of PMK-1 is increased on oxidative stress ${ }^{14}$ and $f l r-4$ mutant worms have higher phospho-PMK-1 on E. coli HT115 bacteria $^{70}$. Although the phosphorylation of PMK-1 has not been directly elucidated on challenge with pathogenic bacteria, susceptible worms have lower amount of pPMK-167,68. In this study, we show that the p38-MAPK pathway is required for both genetic and non-genetic modes of DR, and in case of genetic DR, the pathway is activated and pPMK-1 levels are higher. In contrast to these observations, a recently published study by $\mathrm{Wu}$ et al. showed that although non-genetic modes of DR require p38-MAPK components, the phospho-PMK-1 levels are reduced under this condition ${ }^{69}$. These differences in observations may be attributed to the mode of DR implementation as well as the time-frame when the PPMK-1 westerns were performed. Interestingly, the DR paradigms used in our study that are dependent on the p38-MAPK, also show dependency on the FOXA transcription factor PHA-4 and not the FOXO transcription factor DAF-16 $3,19,21$. On the other hand, the DR paradigm used in $\mathrm{Wu}$ et al. is dependent on FOXO/DAF-16. While these studies underscore the important contribution of the p38-MAPK pathway in DR-induced longevity, it will be important to extensively elucidate the underlying mechanisms in much greater details.

In this context, it may also be noted that unlike $d r l-1$ and eat2 genetic models, we did not find a requirement of p38-MAPK pathway in 2-DOG-mediated life-span extension. Different DR models are known to have distinct as well as complex overlapping mechanisms ${ }^{71}$. For example, in $d r l-1$ and eat- 2 models, ROS is inherently low, while 2-DOG-mediated life-span extension is mediated via the hormetic effects of $\operatorname{ROS}^{3,22}$ Despite these differences, $d r l-1 \mathrm{KD}$ worms, eat-2 mutants as 
well as 2-DOG fed-DR models seems to have a similar downstream NHR-49-mediated metabolic shift that is required for life-span extension ${ }^{3,72}$. How or when DR is initiated may play an important role in activating the p38-MAPK pathway and downstream cytoprotective genes.

\section{Methods}

Strain maintenance. Unless mentioned otherwise, all strains were obtained from CGC Minnesota and maintained at $20^{\circ} \mathrm{C}$ on standard Nematode Growth Media (NGM) seeded with Escherichia coli (OP50). The E. coli bacteria were grown overnight in Luria Bertani (LB) media at $37^{\circ} \mathrm{C}$, and 200 or $1000 \mu \mathrm{l}$ of the culture was seeded on 60 or $90 \mathrm{~mm}$ NGM agar plates, respectively. The plates were set at room temperature for 2-3 days to allow the bacteria to grow. For all RNAi assays, the RNAi was initiated from eggs or synchronized L1s, unless mentioned explicitly. The strains used in the study are: N2 Bristol as wild-type, VC390 nsy-1(ok593) II, KU4 sek-1(km4) X, KU25 pmk-1(km25) IV, VC8 jnk-1(gk7) IV, DA465 eat-2 (ad465) II, DA1116 eat-2(ad1116) II, CY573 bvIs5[cyp-35B1p::GFP+gcy-7p::GFP], BX26 fat-2(wa17) IV, BX156 fat-6(tm331) IV;fat-7(wa36) V, DA2123 adIs2122 [lgg1p::GFP::lgg-1+rol-6(su1006)], fat-2(tm789) IV. The strains eat-2(ad465);fat-2 (tm789), eat-2(ad465) II;sek-1(km4) X, and sek-1(km4);adIs2122 were generated in the lab.

RNAi lifespan. For performing life-span analysis, NGM RNAi plates $(60-\mathrm{mm}$ diameter) were prepared by supplementing NGM with $100 \mu \mathrm{g} / \mathrm{ml}$ ampicillin and $2 \mathrm{mM}$ isopropyl $\beta$-d-1-thiogalactopyranoside (IPTG). RNAi bacteria were grown overnight at $37^{\circ} \mathrm{C}$ in LB media containing $100 \mu \mathrm{g} / \mathrm{ml}$ ampicillin and $12.5 \mu \mathrm{g} / \mathrm{ml}$ tetracycline. The cultures were diluted next day $(1: 100 \mathrm{v} / \mathrm{v})$ in $\mathrm{LB}$ containing $100 \mu \mathrm{g} / \mathrm{ml}$ ampicillin and grown at $37^{\circ} \mathrm{C}$ till an $\mathrm{OD}_{600}$ of 0.6 was attained. The bacterial pellets were then resuspended in $(1: 10 \mathrm{v} / \mathrm{v}) 1 \mathrm{X}$ M9 buffer containing $1 \mathrm{mM}$ IPTG and $100 \mu \mathrm{g} / \mathrm{ml}$ ampicillin. About $200 \mu \mathrm{l}$ bacterial suspension was plated on the NGM RNAi plates and allowed to dry for 1 day.

Synchronized population of worms was obtained by sodium hypochlorite treatment of gravid adult hermaphrodite and eggs were allowed to hatch and grow on plates containing the respective RNAi bacteria. For synchronizing L1s, the eggs were hatched in 1X M9 for $16 \mathrm{~h}$. Day-1 adult worms were then transferred to respective RNAi plates overlaid with 5-fluorodeoxyuridine (FUDR; final concentration of $100 \mu \mathrm{g} / \mathrm{ml})^{73}$. Worms were scored as dead or alive by gently tapping them with a platinum wire every $2-3$ days. Sick worms showing vulval bursting or worms that crawled to the sides/lid of the plates were censored. The fat-2 mutants have been reported to show delayed development at $20^{\circ} \mathrm{C}^{27}$, thus all the life spans involving fat-2 mutants were performed at $25^{\circ} \mathrm{C}$.

2-DOG lifespan. For performing 2-DOG lifespan, NGM agar was supplemented with 2-DOG (Sigma-Aldrich, D8375) to a final concentration of $5 \mathrm{mM}$. Synchronized egg population (100-150) obtained by sodium hypochlorite treatment of gravid worms grown on E. coli OP50 was transferred to control RNAi plates without 2-DOG. At young adult (YA) stage, approximately 50 worms were transferred to the 2-DOG-containing or control plates, both overlaid with FUDR, in three technical replicates. Worms were then scored as live or dead every alternate day. Sick worms with ruptured vulva rupture were censored from the population.

Bacterial dilution-induced DR (BDR) assay. The BDR assay was performed by preparing E. coli OP50-L4440 (for Fig. 2c) or HT115-L4440 (Fig. 5e) BDR media as described below. Using a platinum loop, E. coli was streaked on a LB agar plate and incubated at $37^{\circ} \mathrm{C}$ for $12 \mathrm{~h}$. From the plate, single bacterial colony was used to inoculate $200 \mathrm{ml} \mathrm{LB}$ in a 2-litre flask. Bacteria were allowed to grow at $37^{\circ} \mathrm{C}$ for $12 \mathrm{~h}$ in an incubator shaker (Innova 42 incubator shaker, New Brunswick Scientific, Edison, NJ, USA) at $250 \mathrm{rpm}$. The bacterial cells were collected by centrifugation at $3215 \times g, 4^{\circ} \mathrm{C}$ for $10 \mathrm{~min}$ and resuspended in S-basal-cholesterol-antibiotics solution (cholesterol $5 \mu \mathrm{g} / \mathrm{ml}$, carbenicillin $50 \mu \mathrm{g} / \mathrm{ml}$, tetracycline $1 \mu \mathrm{g} / \mathrm{ml}$, kanamycin $10 \mu \mathrm{g} / \mathrm{ml})$. The desired bacterial dilution was measured by further diluting cells in Sbasal-cholesterol-antibiotics solution and measuring $\mathrm{OD}_{600}$. These diluted bacterial solutions were stored at $4{ }^{\circ} \mathrm{C}$ for a maximum of 2 weeks. Synchronized egg population obtained by sodium hypochlorite treatment of well-fed gravid adult worms were allowed to grow on a $90 \mathrm{~mm}$ NGM plate seeded with OP50 bacteria till young adult age. At this point, FUDR $(100 \mu \mathrm{g} / \mathrm{ml})$ was added on plates to prevent any progeny development. Post $24 \mathrm{~h}$, worms were transferred to 12 -well cell-culture plate (30 worms/well) containing $1 \mathrm{ml}$ of S-basal-cholesterol-antibiotics solution with FUDR $(100 \mu \mathrm{g} / \mathrm{ml})$. The plate was kept on a shaker maintained at $20^{\circ} \mathrm{C}$ for $1 \mathrm{~h}$ to remove adhering bacteria. During this time, diluted bacterial suspension for the lifespan were added to the 12 -well cell-culture plates $(1 \mathrm{ml}$ solution/well along with FUDR at $100 \mu \mathrm{g} / \mathrm{ml}$ ). After $1 \mathrm{~h}, 10-12$ worms/well were moved from the S-basal to the diluted bacterial suspension using a glass pipette connected to a P200 pipette. Worms were moved to fresh bacterial solutions with respective ODs every 3-4 days and scored for movement by prodding using a platinum wire. FUDR supplementation in diluted bacterial suspension was stopped after 8 days. Worms that did not respond to gentle prodding with a worm pick were scored as dead and removed. During experiments, plates were maintained at $20^{\circ} \mathrm{C}$ for sek- $1(\mathrm{~km} 4)$ and $25^{\circ} \mathrm{C}$ for fat-2(wa17) experiments, in an incubator shaker (Innova 42 incubator shaker, New Brunswick Scientific, Edison, NJ, USA) at rotation speed of $100 \mathrm{rpm}$. Range of $\mathrm{OD}_{600}$ used: $3.0,1.0,0.5,0.25,0.125$, and 0.0156 .

Lethality assay. The pos- 1 gene codes for a CCCH-type zinc finger protein that is required for specification of germ cells, intestine, pharynx, and hypodermis ${ }^{74}$; knocking it down leads to larval lethality. Wild-type, nsy-1, sek-1, and pmk-1 L4 animals were picked and maintained on control or pos-1 RNAi-seeded plate till day-1 of adulthood and sacrificed. Eggs laid on the RNAi plates were scored for hatching. Percentage of eggs that failed to hatch or arrested early in development (embryonic lethal) were plotted on $\mathrm{X}$-axis with $\mathrm{Y}$-axis indicating the genotype.

Quantification of fat content by Oil Red $\mathbf{O}$ staining. Fat storage was determined in fixed worms using Oil Red $\mathrm{O}^{24}$. Oil Red O stain was prepared as a $5 \mathrm{mg} / \mathrm{ml}$ stock in isopropanol and equilibrated on a rocker-shaker for 6-7 days. The working solution of Oil Red O was prepared by diluting the equilibrated stock to $60 \%$ with water. Stock was mixed thoroughly and filtered using a $0.22 \mu \mathrm{m}$ filter to remove any particles. Gravid adult worms were treated with sodium hypochlorite to obtain eggs which were grown on respective RNAi plates till L4-YA stage. The worms were washed in 1X M9 to remove any attached bacteria and resuspended in $120 \mu \mathrm{l} 1 \mathrm{X}$ PBS. To this an equal volume of 2X MRWB buffer $(160 \mathrm{mM} \mathrm{KCl}, 40 \mathrm{mM} \mathrm{NaCl}$, $14 \mathrm{mM} \mathrm{Na} 2$ EGTA, PIPES pH 7.4, $1 \mathrm{mM}$ spermidine, $0.4 \mathrm{mM}$ spermine, $2 \%$ Paraformaldehyde, and $0.2 \%$ beta-mercaptoethanol) was added and incubated by shaking for $45 \mathrm{~min}$. The worms were pelleted and washed with 1X PBS. The working solution of Oil Red $\mathrm{O}$ was added to the fixed worms and incubated for $1 \mathrm{~h}$ on a shaker at room temperature. Following staining, worms were washed thrice with $1 \mathrm{X}$ PBS and mounted on $2 \%$ agarose slides for visualization using an AxioImager M2 microscope (Carl Zeiss, Germany) fitted with an Axiocam MRc camera. NIH ImageJ software $1.52 \mathrm{a}$ was used to quantify fat stores in the worms.

Oxygen consumption rate (OCR). Synchronized population of worms was grown at $20^{\circ} \mathrm{C}$ till YA stage on RNAi- or OP50-seeded plates from egg onwards. From this population, 400 YA worms were picked into 1X M9 buffer and washed thrice to remove any attached bacteria before placing it in the respiratory chambers of Oxygraph-2k (A\&B) (Oroboros Instruments, Innsbruck, Austria). OCR was monitored in real-time as decline in $\mathrm{O}_{2}$ saturation in the two chambers. Data, obtained with the inbuilt software Datlab 7, is plotted as the rate of decline in $\mathrm{O}_{2}$ consumption (respiration rate) over $10 \mathrm{~min}$ of time interval.

ROS measurement. For measuring intracellular ROS levels, synchronized YA worms were collected and washed thrice in 1X M9 buffer. Worm extracts were prepared by flash freezing worm pellets in liquid nitrogen and freeze-thawing for three cycles, followed by sonication (setting of 30 amplitudes, 12 cycles of $1 \mathrm{~s}$ pulse on/off, 5-8 times using Misonix Ultrasonic processor 4000) in 1X PBS. The worm extract was centrifuged at $20,000 \times g$ for $15 \mathrm{~min}$ at $4^{\circ} \mathrm{C}$, and the protein concentration of the supernatant was determined using Bradford protein estimation kit (Bio-Rad, USA). Supernatant containing $5 \mu \mathrm{g}$ of protein was pre-incubated with $50 \mu \mathrm{M}$ of 2,7-dichlorofluorescein diacetate (DCFDA, Molecular Probes, USA) in $100 \mu \mathrm{L}$ of $1 \mathrm{X} \mathrm{PBS}$ at $37^{\circ} \mathrm{C}$ for $1 \mathrm{~h}$. Fluorescence intensity was measured in FLUOstar Omega (BMG Labtech, USA) every $10 \mathrm{~min}$ for $1 \mathrm{~h}$ at excitation wavelength $485 \mathrm{~nm}$ and emission wavelength $520 \mathrm{~nm}$.

Pharyngeal pumping. Pharyngeal pumping was counted in L4 worms grown on control RNAi from hatching. A 1-min video of each worm was taken using Axiocam MRm (Carl Zeiss, Germany) camera attached to M205FA microscope (Leica, Germany). The video was slowed down and pharyngeal pumping was counted for this period.

Autophagy analysis. Transgenic reporter strains expressing GFP-tagged LGG-1 in wild-type, eat-2(ad465), or sek-1(km4) mutant background were grown at $20^{\circ} \mathrm{C}$ on control, sek-1, or drl-1 RNAi from egg onward. The L3 larval stage worms were picked onto $2 \%$ agarose slides and paralyzed using $20 \mathrm{mM}$ of sodium azide. Worms were imaged for LGG-1:GFP puncta in their hypodermal seam cells using AxioImager M2 microscope (Carl Zeiss, Germany) fitted with Axiocam MRm at a magnification of $\times 630$. Scoring of 3-10 seam cells were performed for each of the worms examined. The autophagic events were quantified and plotted as the number of puncta per seam cell by averaging the total number of puncta over all the seam cells counted.

Total RNA isolation and quantitative real-time PCR. Synchronized L1 worms grown till YA stage on control or $d r l-1$ RNAi plates were exposed to FUDR to prevent any progeny growth. Post $24 \mathrm{~h}$, worms were collected, washed thrice in $1 \mathrm{X}$ M9 buffer and flash-frozen in Trizol (Invitrogen, USA). The worms in Trizol were passed through a freeze-thaw cycle and lysed by vigorous vortexing. RNA was purified by phenol:chloroform:isoamylalcohol extraction followed by ethanol precipitation. The concentration of the RNA was determined using NanoDrop 2000 
(Thermo Scientific, USA). The quality of the ribosomal $28 \mathrm{~s}$ and $18 \mathrm{~s}$ on an agarose gel was used as a measure of integrity. Around $2.5 \mu \mathrm{g}$ of RNA was used for cDNA synthesis using Superscript III Reverse Transcriptase (Invitrogen, USA). For determining mRNA expression levels, quantitative real-time PCR (qRT-PCR) was performed using the DyNAmo Flash SYBR Green mastermix (Thermo Scientific, USA) and Realplex PCR system (Eppendorf, USA), according to the manufacturer's specifications. Relative mRNA expression for target genes was determined after normalizing the data to actin.

XDP reporter imaging and analysis. The transgenic C. elegans strain carrying a $c y p-35 B 1$ promoter fused to the green fluorescent protein was used to visualize the expression of cyp-35B1 upon drl-1 knockdown. CY573 (bvIs5 [cyp-35B1p::GFP + $g c y-7 p:: G F P])$ worms were grown at $20^{\circ} \mathrm{C}$ on standard NGM plates seeded with $E$. coli (OP50). Synchronized population of eggs were allowed to hatch and grow till L2 larval stage on plates containing the control or $d r l-1$ RNAi-seeded bacteria. Post L2, worms were transferred to control, $d r l-1$ or sek-1 RNAi plates (Fig. $4 \mathrm{~b}$ ). Worms were imaged using Zeiss Imager M2 at L4-YA stage. Around 50 adult worms were picked onto $2 \%$ agarose slides and paralyzed using $50 \mathrm{mM}$ of sodium azide in $1 \mathrm{X}$ M9. Worms were imaged using an AxioImager M2 microscope at a magnification of $\times 100$, which was kept similar throughout all conditions. Over 30 transgenic worms per condition were imaged and analyzed. For fat-2(wa17);Pcyp-35B1::gfp, the worms were maintained and the experiments performed at $25^{\circ} \mathrm{C}$.

Fatty acid analysis using GC-MS. To measure fatty acid composition, $\sim 1000$ young adult stage worms (containing $0-8$ embryos) were washed from feeding plates with water on ice and washed once to remove residual bacteria. After settling on ice again, as much water as possible was removed $(\sim 90 \%)$. Fatty acids were converted to methyl esters for analysis as previously described ${ }^{75}$. The worm suspensions were incubated for $1 \mathrm{~h}$ at $70^{\circ} \mathrm{C}$ in $2 \mathrm{ml}$ of $2.5 \%$ sulfuric acid in methanol. Following incubation, the reactions were stopped by adding $1 \mathrm{ml}$ of water and then mixed thoroughly with $200 \mu \mathrm{l}$ of hexane to extract the resulting fatty acid methyl esters. We measured relative amount of fatty acid methyl esters by injecting $2 \mu \mathrm{l}$ of the hexane layer onto an Agilent 7890 GC/5975C MS in scanning ion mode.

External supplementation assay. For external supplementation of PUFAs ${ }^{76}$, NGM agar media containing $0.1 \%$ Tergitol (NP-40) was autoclaved and allowed to partially cool till the temperature reached $65^{\circ} \mathrm{C}$. To this, $0.3 \mathrm{mM}$ linoleic acid (Catalog no. 90150), $0.5 \mathrm{mM}$ eicasopentaenoic acid (Catalog no. 90110) (Cayman Chemical, USA) or ethanol control were added with constant stirring at around $65^{\circ} \mathrm{C}$. After pouring, plates were kept for drying in the dark for 2 days at room temperature. E. coli OP50-RNAi strain transformed with control or sek-1 RNAi plasmid were seeded on these plates. Plates were allowed to dry for 3 days at a temperature of $20^{\circ} \mathrm{C}$. Synchronized population of eggs obtained from sodium hypochlorite treatment of gravid adult CY573 worms (bvIs5[cyp-35B1p::GFP+gcy$7 p:: G F P])$ were allowed to hatch and grown till YA on different fatty acid supplemented plates at $20^{\circ} \mathrm{C}$. About $36-40 \mathrm{~h}$ after YA, worms were imaged using Zeiss Imager M2 as discussed above. At the same time, 50 worms were collected in $1 \mathrm{X}$ M9 buffer, washed once with 1X M9 and denatured in 1X Lamelli's buffer to be used for phospho-western analysis. For oleic acid (OA; catalog no. 01008-1G; Merck, USA), palmitic acid (PA; catalog no. P0500-10G; Merck, USA), stearic acid (SA; catalog no. S4751-10G; Merck, USA), and the fatty acids were supplemented to the bacterial pellet at a concentration of $4.8 \mathrm{mM}$, respectively. Worms were imaged 24-28 h post YA. For life-span analysis (Fig. 7a, b), supplementation was done from L1 stage.

Statistical analysis. All survival graphs were plotted using GraphPad Prism 8 (GraphPad Software, Inc., La Jolla, CA). All the statistical analysis to measure $P$ values between survival curves was performed using Log-rank (Mantel-Cox) test through online software OASIS 1.0 [http://sbi.postech.ac.kr/oasis]. Summary of life span containing mean lifespan \pm SEM, number of animals $(n)$ and $P$-value is provided in Supplementary Table 1. Two-way ANOVA as well as unpaired twotailed $t$ test was used in case of BDR life spans as indicated in the figure legends. Two-way ANOVA was used for ROS (Fig. 3c) and autophagy (Fig. 3e, Supplementary Fig. 3b) measurements. Unpaired two-tailed Student's $t$ test was used for Figs. 3d, 5f, 6a, Supplementary Figs. 1a, b, 2a-c, 3a, 6).

Western blot analysis. For each sample, 50 worms were picked in $1 \mathrm{X}$ M9 buffer, washed twice and collected in 1X SDS lamellae buffer. It was then boiled for $10 \mathrm{~min}$ and resolved by $10 \%$ SDS-PAGE. After complete run, proteins were transferred onto PVDF membrane (Millipore, Billerica, MA) at $110 \mathrm{~V}$ for $1 \mathrm{~h}$ at $4{ }^{\circ} \mathrm{C}$ in chilled transfer buffer. Following transfer, the membrane was incubated with $5 \% \mathrm{w} / \mathrm{v}$ bovine serum albumin (BSA) prepared in 0.1\% TBST for blocking. The membrane was blocked for $1 \mathrm{~h}$ at room temperature. Subsequently, primary antibody was added in a 1:5000 dilution, prepared in $0.1 \%$ TBST and incubated overnight $4{ }^{\circ} \mathrm{C}$ in a rocker-shaker. Next day, after $16 \mathrm{~h}$ of incubation, excess primary antibody was washed off with three washes of $0.1 \%$ TBST, each wash for $5 \mathrm{~min}$ at RT on a rocker-shaker. Secondary antibody (Abcam, Cat no.: ab6721) at a dilution of 1:5000 in $0.1 \%$ TBST was added to the blot and incubated for $1 \mathrm{~h}$ at RT with rocking. Again, the blot was washed by $0.1 \%$ TBST three times, each for $5 \mathrm{~min}$, at
RT on a rocker-shaker. Then, the blot was developed using an ECL reagent (Millipore, USA), according to manufacturer's instructions, to detect antibody binding to specific protein bands on the membrane. Uncropped blots are provided in the Source data file.

Antibody/Supplier name/Catalog no./lot number

1. Phospho-p38-MAPK (Thr180/Tyr182) (3D7) Rabbit mAb/Cell Signaling Technology/9215S/7

2. p38-MAPK Antibody/Cell Signaling Technology/9212S/26

3. $\beta$-Actin Antibody/Cell Signaling Technology/4967L/7

4. Goat anti-rabbit IgG H\&L (HRP) Secondary antibody/Abcam/ab6721

Reporting summary. Further information on research design is available in the Nature Research Reporting Summary linked to this article.

\section{Data availability}

Source data are provided with this paper. All other relevant data are available in the article, supplementary information, or from the corresponding author upon reasonable request. Life-span data used in Figs. 1, 2, 5, 7 and Supplementary Fig. 1 are presented in Supplementary Table 1, along with an independent biological replicate. Source data are provided with this paper.

Received: 27 August 2019; Accepted: 2 September 2020; Published online: 25 September 2020

\section{References}

1. Speakman, J. R. \& Mitchell, S. E. Caloric restriction. Mol. Asp. Med. 32 159-221 (2011).

2. Katewa, S. D. et al. Intramyocellular fatty-acid metabolism plays a critical role in mediating responses to dietary restriction in Drosophila melanogaster. Cell Metab. 16, 97-103 (2012)

3. Chamoli, M., Singh, A., Malik, Y. \& Mukhopadhyay, A. A novel kinase regulates dietary restriction-mediated longevity in Caenorhabditis elegans. Aging Cell 13, 641-655 (2014).

4. Chen, D. et al. Tissue-specific regulation of SIRT1 by calorie restriction. Genes Dev. 22, 1753-1757 (2008).

5. Cao, S. X., Dhahbi, J. M., Mote, P. L. \& Spindler, S. R. Genomic profiling of short- and long-term caloric restriction effects in the liver of aging mice. Proc. Natl Acad. Sci. USA 98, 10630-10635 (2001).

6. Anderson, R. M. \& Weindruch, R. Metabolic reprogramming in dietary restriction. Interdiscip. Top. Gerontol. 35, 18-38 (2007).

7. Anderson, R. M. \& Weindruch, R. Metabolic reprogramming, caloric restriction and aging. Trends Endocrinol. Metab. 21, 134-141 (2010).

8. Cowan, K. J. \& Storey, K. B. Mitogen-activated protein kinases: new signaling pathways functioning in cellular responses to environmental stress. J. Exp. Biol. 206, 1107-1115 (2003).

9. Zarubin, T. \& Han, J. Activation and signaling of the p38 MAP kinase pathway. Cell Res. 15, 11 (2005).

10. Krishna, M. \& Narang, H. The complexity of mitogen-activated protein kinases (MAPKs) made simple. Cell Mol. Life Sci. 65, 3525-3544 (2008).

11. Eun Kyung, K. \& Eui-Ju, C. Pathological roles of MAPK signaling pathways in human diseases. Biochim. Biophys. Acta Mol. Basis Dis. 1802, 396-405 (2010).

12. Alan, J. W. A central role for $\mathrm{p} 38-\mathrm{MAPK}$ in the early transcriptional response to stress. BMC Biol. 8, 47 (2010).

13. Millet, A. C. \& Ewbank, J. J. Immunity in Caenorhabditis elegans. Curr. Opin. Immunol. 16, 4-9 (2004).

14. Inoue, $\mathrm{H}$. et al. The $C$. elegans $\mathrm{p} 38-\mathrm{MAPK}$ pathway regulates nuclear localization of the transcription factor SKN-1 in oxidative stress response. Genes Dev. 19, 2278-2283 (2005).

15. Troemel, E. R. et al. p38-MAPK regulates expression of immune response genes and contributes to longevity in C. elegans. PLoS Genet. 2, e183 (2006).

16. Shivers, R. P. et al. Phosphorylation of the conserved transcription factor ATF-7 by PMK-1 p38-MAPK regulates innate immunity in Caenorhabditis elegans. PLoS Genet. 6, e1000892 (2010).

17. Mertenskotter, A., Keshet, A., Gerke, P. \& Paul, R. J. The p38-MAPK PMK 1 shows heat-induced nuclear translocation, supports chaperone expression, and affects the heat tolerance of Caenorhabditis elegans. Cell Stress Chaperones 18, 293-306 (2013)

18. Zarse, K. et al. Impaired insulin/IGF1 signaling extends life span by promoting mitochondrial L-proline catabolism to induce a transient ROS signal. Cell Metab. 15, 451-465 (2012).

19. Lakowski, B. \& Hekimi, S. The genetics of caloric restriction in Caenorhabditi elegans. Proc. Natl Acad. Sci. USA 95, 13091-13096 (1998). 
20. Mair, W., Panowski, S. H., Shaw, R. J. \& Dillin, A. Optimizing dietary restriction for genetic epistasis analysis and gene discovery in C. elegans. PLoS ONE 4, e4535 (2009).

21. Panowski, S. H., Wolff, S., Aguilaniu, H., Durieux, J. \& Dillin, A. PHA-4/Foxa mediates diet-restriction-induced longevity of C. elegans. Nature 447, 550-555 (2007).

22. Schulz, T. J. et al. Glucose restriction extends Caenorhabditis elegans life span by inducing mitochondrial respiration and increasing oxidative stress. Cell Metab. 6, 280-293 (2007).

23. Van Gilst, M. R., Hadjivassiliou, H., Jolly, A. \& Yamamoto, K. R. Nuclear hormone receptor NHR-49 controls fat consumption and fatty acid composition in C. elegans. PLoS Biol. 3, e53 (2005).

24. O’Rourke, E. J., Kuballa, P., Xavier, R. \& Ruvkun, G. omega-6 Polyunsaturated fatty acids extend life span through the activation of autophagy. Genes Dev. 27, 429-440 (2013).

25. Nandakumar, M. \& Tan, M. W. Gamma-linolenic and stearidonic acids are required for basal immunity in Caenorhabditis elegans through their effects on p38 MAP kinase activity. PLoS Genet. 4, e1000273 (2008).

26. Vrablik, T. L. \& Watts, J. L. Polyunsaturated fatty acid derived signaling in reproduction and development: insights from Caenorhabditis elegans and Drosophila melanogaster. Mol. Reprod. Dev. 80, 244-259 (2013).

27. Watts, J. L. \& Browse, J. Genetic dissection of polyunsaturated fatty acid synthesis in Caenorhabditis elegans. Proc. Natl Acad. Sci. USA 99, 5854-5859 (2002).

28. Brock, T. J., Browse, J. \& Watts, J. L. Fatty acid desaturation and the regulation of adiposity in Caenorhabditis elegans. Genetics 176, 865-875 (2007).

29. Watts, J. L. \& Ristow, M. Liquid and carbohydrate metabolism in Caenorhabditis elegans. Genetics 207, 413-446 (2017).

30. Jove, M. et al. Caloric restriction reveals a metabolomic and lipidomic signature in liver of male mice. Aging Cell 13, 828-837 (2014).

31. Weaver, K. L., Ivester, P. \& Chilton, F. Influence of aging and caloric restriction on the levels of polyunsaturated fatty acids involved in inflammation and gene expression. FASEB J. 20, A140-A140 (2006).

32. Steinbaugh, M. J., Sun, L. Y., Bartke, A. \& Miller, R. A. Activation of genes involved in xenobiotic metabolism is a shared signature of mouse models with extended lifespan. Am. J. Physiol. Endocrinol. Metab. 303, E488-E495 (2012).

33. Wen, H. et al. Enhanced phase II detoxification contributes to beneficial effects of dietary restriction as revealed by multi-platform metabolomics studies. Mol. Cell. Proteom. 12, 575-586 (2013).

34. Van Gilst, M. R., Hadjivassiliou, H. \& Yamamoto, K. R. A Caenorhabditis elegans nutrient response system partially dependent on nuclear receptor NHR-49. Proc. Natl Acad. Sci. USA 102, 13496-13501 (2005).

35. Mobbs, C. V. et al. Secrets of the lac operon. Glucose hysteresis as a mechanism in dietary restriction, aging and disease. Interdiscip. Top. Gerontol. 35, 39-68 (2007).

36. Guarente, L. Mitochondria-a nexus for aging, calorie restriction, and sirtuins? Cell 132, 171-176 (2008).

37. Pratt, D. A., Tallman, K. A. \& Porter, N. A. Free radical oxidation of polyunsaturated lipids: new mechanistic insights and the development of peroxyl radical clocks. Acc. Chem. Res. 44, 458-467 (2011).

38. Yang, W. S. et al. Peroxidation of polyunsaturated fatty acids by lipoxygenases drives ferroptosis. Proc. Natl Acad. Sci. USA 113, E4966-E4975 (2016).

39. Ayala, A., Munoz, M. F. \& Arguelles, S. Lipid peroxidation: production, metabolism, and signaling mechanisms of malondialdehyde and 4-hydroxy-2nonenal. Oxid. Med. Cell. Longev. 2014, 360438 (2014).

40. Awada, M. et al. Dietary oxidized n-3 PUFA induce oxidative stress and inflammation: role of intestinal absorption of 4-HHE and reactivity in intestinal cells. J. Lipid Res. 53, 2069-2080 (2012).

41. Beaudoin-Chabot, C. et al. Deuterated polyunsaturated fatty acids reduce oxidative stress and extend the lifespan of C. elegans. Front. Physiol. 10, 641 (2019).

42. Michalak, A., Mosinska, P. \& Fichna, J. Polyunsaturated fatty acids and their derivatives: therapeutic value for inflammatory, functional gastrointestinal disorders, and colorectal cancer. Front. Pharmacol. 7, 459 (2016).

43. Gerisch, B. \& Antebi, A. Hormonal signals produced by DAF-9/cytochrome P450 regulate $C$. elegans dauer diapause in response to environmental cues. Development 131, 1765-1776 (2004).

44. Gerisch, B. et al. A bile acid-like steroid modulates Caenorhabditis elegans lifespan through nuclear receptor signaling. Proc. Natl Acad. Sci. USA 104, 5014-5019 (2007).

45. Jia, K., Albert, P. S. \& Riddle, D. L. DAF-9, a cytochrome P450 regulating C. elegans larval development and adult longevity. Development 129, 221-231 (2002).

46. Ashrafi, K. et al. Genome-wide RNAi analysis of Caenorhabditis elegans fat regulatory genes. Nature 421, 268-272 (2003).

47. Kubagawa, H. M. et al. Oocyte signals derived from polyunsaturated fatty acids control sperm recruitment in vivo. Nat. Cell Biol. 8, 1143-1148 (2006).
48. Kulas, J., Schmidt, C., Rothe, M., Schunck, W. H. \& Menzel, R. Cytochrome P450-dependent metabolism of eicosapentaenoic acid in the nematode Caenorhabditis elegans. Arch. Biochem. Biophys. 472, 65-75 (2008).

49. Papsdorf, K. \& Brunet, A. Linking lipid metabolism to chromatin regulation in aging. Trends Cell Biol. 29, 97-116 (2019).

50. Talukdar, I., Szeszel-Fedorowicz, W. \& Salati, L. M. Arachidonic acid inhibits the insulin induction of glucose-6-phosphate dehydrogenase via p38 MAP kinase. J. Biol. Chem. 280, 40660-40667 (2005).

51. Evans, J. et al. Arachidonic acid induces brain endothelial cell apoptosis via p38-MAPK and intracellular calcium signaling. Microvasc. Res. 98, 145-158 (2015).

52. Gu, L. Y., Qiu, L. W., Chen, X. F., Lu, L. \& Mei, Z. C. Oleic acid-induced hepatic steatosis is coupled with downregulation of aquaporin 3 and upregulation of aquaporin 9 via activation of p38 signaling. Horm. Metab. Res. 47, 259-264 (2015).

53. Yamasaki, M. et al. Oleic acid prevents apoptotic cell death induced by trans10, cis12 isomer of conjugated linoleic acid via p38 MAP kinase dependent pathway. Vitr. Cell. Dev. Biol. Anim. 44, 290-294 (2008).

54. Hennig, B. et al. Linoleic acid induces proinflammatory events in vascular endothelial cells via activation of PI3K/Akt and ERK1/2 signaling. J. Nutr. Biochem. 17, 766-772 (2006).

55. Collins, Q. F., Xiong, Y., Lupo, E. G. Jr., Liu, H. Y. \& Cao, W. p38 Mitogen activated protein kinase mediates free fatty acid-induced gluconeogenesis in hepatocytes. J. Biol. Chem. 281, 24336-24344 (2006).

56. Caires, R. et al. Omega-3 fatty acids modulate TRPV4 function through plasma membrane remodeling. Cell Rep. 21, 246-258 (2017).

57. Kahn-Kirby, A. H. et al. Specific polyunsaturated fatty acids drive TRPVdependent sensory signaling in vivo. Cell 119, 889-900 (2004).

58. Chaudhuri, J. et al. A Caenorhabditis elegans model elucidates a conserved role for TRPA1-Nrf signaling in reactive alpha-dicarbonyl detoxification. Curr. Biol. 26, 3014-3025 (2016).

59. Tanaka, A. et al. Polyunsaturated fatty acids induce ovarian cancer cell death through ROS-dependent MAP kinase activation. Biochem. Biophys. Res. Commun. 493, 468-473 (2017).

60. Watts, J. L. \& Browse, J. Dietary manipulation implicates lipid signaling in the regulation of germ cell maintenance in C. elegans. Dev. Biol. 292, 381-392 (2006).

61. Webster, C. M., Deline, M. L. \& Watts, J. L. Stress response pathways protect germ cells from omega- 6 polyunsaturated fatty acid-mediated toxicity in Caenorhabditis elegans. Dev. Biol. 373, 14-25 (2013).

62. Shmookler Reis, R. J. et al. Modulation of lipid biosynthesis contributes to stress resistance and longevity of C. elegans mutants. Aging 3, 125-147 (2011).

63. Sugawara, S., Honma, T., Ito, J., Kijima, R. \& Tsuduki, T. Fish oil changes the lifespan of Caenorhabditis elegans via lipid peroxidation. J. Clin. Biochem. Nutr. 52, 139-145 (2013).

64. Hamilton, L. K. et al. Aberrant lipid metabolism in the forebrain niche suppresses adult neural stem cell proliferation in an animal model of Alzheimer's disease. Cell Stem Cell 17, 397-411 (2015).

65. Chuang, C. F. \& Bargmann, C. I. A Toll-interleukin 1 repeat protein at the synapse specifies asymmetric odorant receptor expression via ASK1 MAPKKK signaling. Genes Dev. 19, 270-281 (2005).

66. Sagasti, A. et al. The CaMKII UNC-43 activates the MAPKKK NSY-1 to execute a lateral signaling decision required for asymmetric olfactory neuron fates. Cell 105, 221-232 (2001).

67. Youngman, M. J., Rogers, Z. N. \& Kim, D. H. A decline in p38-MAPK signaling underlies immunosenescence in Caenorhabditis elegans. PLoS Genet. 7, e1002082 (2011).

68. Kim, D. H. et al. A conserved p38 MAP kinase pathway in Caenorhabditis elegans innate immunity. Science 297, 623-626 (2002).

69. $\mathrm{Wu}, \mathrm{Z}$. et al. Dietary restriction extends lifespan through metabolic regulation of innate immunity. Cell Metab. 29, 1192-1205 e1198 (2019).

70. Verma, S., Jagtap, U., Goyala, A. \& Mukhopadhyay, A. A novel gene-diet pair modulates C. elegans aging. PLoS Genet. 14, e1007608-e1007608 (2018).

71. Greer, E. L. \& Brunet, A. Different dietary restriction regimens extend lifespan by both independent and overlapping genetic pathways in C. elegans. Aging Cell 8, 113-127 (2009).

72. Marcellino, B. K., Ekasumara, N. \& Mobbs, C. V. Dietary restriction and glycolytic inhibition reduce proteotoxicity and extend lifespan via NHR-49. J. Curr. Neurobiol. 9, 1-7 (2018).

73. Hosono, R., Mitsui, Y., Sato, Y., Aizawa, S. \& Miwa, J. Life span of the wild and mutant nematode Caenorhabditis elegans. Effects of sex, sterilization, and temperature. Exp. Gerontol. 17, 163-172 (1982).

74. Tabara, H. et al. The rde-1 gene, RNA interference, and transposon silencing in C. elegans. Cell 99, 123-132 (1999).

75. Shi, X. et al. Regulation of lipid droplet size and phospholipid composition by stearoyl-CoA desaturase. J. Lipid Res. 54, 2504-2514 (2013). 
76. Deline, M. L., Vrablik, T. L. \& Watts, J. L. Dietary supplementation of polyunsaturated fatty acids in Caenorhabditis elegans. J. Vis. Exp. https://doi. org/10.3791/50879 (2013).

\section{Acknowledgements}

We would like to thank the present and former members of the Molecular Aging lab for their assistance. This project was partly funded by the Ramalingaswami Fellowship (BT/ HRD/35/02/12/2008) and National Bioscience Award for Career Development (BT/ HRD/NBA/38/04/2016) to A.M. We also thank Department of Biotechnology (DBT), Govt. of India, for a generous grant to set up the NGS core facility at the institute. M.C. and S.S.T. were supported by Junior and Senior Research Fellowships from DBT, A.S from Council of Scientific and Industrial Research (CSIR). M.C. is supported by the postdoctoral fellowship from Larry L. Hillblom foundation. The research was supported in part by DBT Grant Nos. BT/PR13720/BAB/10/779/2010 and BT/PR16823/NER/95/ 305/2015, Department of Science and Technology-Science and Engineering Research Board (DST-SERB) Grant No. EMR/2014/000377 and core funding from the National Institute of Immunology. Some strains were provided by the Caenorhabditis Genetics Center, which is funded by National Institute of Health Office of Research Infrastructure Programs (P40 OD010440). The fat-2(tm789) was obtained from National Bioresource Project for the Experimental Animal "Nematode C. elegans", Tokyo, Japan.

\section{Author contributions}

A.M. and M.C. conceptualized the project and wrote the manuscript, while G.J.L. and J.L.W. edited it. M.C., A.G., S.S.T., A.A.S., A.S., and J.L.W. performed experiments and analyzed data. G.J.L., J.L.W., A.A., and A.M. supervised the research.

\section{Competing interests}

The authors declare no competing interests.

\section{Additional information}

Supplementary information is available for this paper at https://doi.org/10.1038/s41467020-18690-4.

Correspondence and requests for materials should be addressed to A.M.

Peer review information Nature Communications thanks Ralph Menzel and the other, anonymous, reviewer(s) for their contribution to the peer review of this work.

Reprints and permission information is available at http://www.nature.com/reprints

Publisher's note Springer Nature remains neutral with regard to jurisdictional claims in published maps and institutional affiliations.

(c) Open Access This article is licensed under a Creative Commons Attribution 4.0 International License, which permits use, sharing, adaptation, distribution and reproduction in any medium or format, as long as you give appropriate credit to the original author(s) and the source, provide a link to the Creative Commons license, and indicate if changes were made. The images or other third party material in this article are included in the article's Creative Commons license, unless indicated otherwise in a credit line to the material. If material is not included in the article's Creative Commons license and your intended use is not permitted by statutory regulation or exceeds the permitted use, you will need to obtain permission directly from the copyright holder. To view a copy of this license, visit http://creativecommons.org/ licenses/by/4.0/.

(c) The Author(s) 2020 